\title{
Analysis of the Effect of Source Capacitance and Inductance on $N$-path Mixers and Filters
}

\author{
Shanthi Pavan, Senior Member, IEEE and Eric Klumperink, Senior Member, IEEE
}

\begin{abstract}
Switch-R-C passive $N$-path mixers and filters enable interference-robust radio receivers with a flexibly programmable center frequency defined by a digital multi-phase clock. The radio frequency ( $R F$ ) range of these circuits is limited by parasitic shunt capacitances, which introduce signal loss and degrade noise figure. Moreover, the linear periodically time varying (LPTV) nature of switch-R-C circuits results in unwanted signal folding which needs to be suppressed by linear time- invariant (LTI) pre-filtering by passive LC filters. This paper analyzes the interaction between capacitive or inductive LTI pre-filtering and an $N$-path mixer or filter, leveraging an analysis technique based on the impulse response of the adjoint network. Previously reported results for an inductive source impedance are derived in a simpler fashion, while providing circuit intuition. Moreover, new results for $N$-path receivers with a shunt capacitor, and a combination of a series inductor and shunt capacitor are derived, as well as design criteria to minimize loss and frequency shifting in the peak response of these circuits.
\end{abstract}

\section{INTRODUCTION}

Reconfigurable radio receivers need tunable filters and linear mixers with strong blocker handling capability [1]. Hard-switched passive mixers using triode-operated MOSFET switches are instrumental in achieving high-linearity while minimizing $1 / f$ noise [2]-[4]. A low-noise transconductance amplifier (LNTA) often precedes the passive mixer to minimize noise figure, realize impedance matching and reduce LO-radiation. The mixer transfer function and noise in this current-driven mode, where the RF-source can be represented by a Norton equivalent, has been extensively analyzed in the literature [5]-[10]. Simple analysis is possible if the baseband impedance $Z_{b b}$ has both a resistive and capacitive component, as shown in Fig. 1. The insight emerging from this analysis is that of frequency-translated filtering: the low-pass baseband impedance $Z_{b b}$ in a zero-IF receiver gets upconverted (frequency translated) by the bi-directionally operating passive mixer to a band-pass impedance at RF. This bandpass filtering reduces the out-of-band signal swing at the LNTA-output, improving linearity. Still, the active devices in the LNTA limit the compression point to less that $0 \mathrm{dBm}[3]$ and $\mathrm{IIP}_{3}$ to 10 $15 \mathrm{dBm}$ [3], [4] in practice, especially due to process spread [11].

Passive switch-RC mixer-first receivers with built-in or explicit $N$-path filtering have recently been proposed [12][17] to improve out-of-band linearity further. The upconverted passive filter in Fig. 1 now filters the antenna signal directly before any active amplification, resulting in reported blocker compression points in excess of $+10 \mathrm{dBm}$ and IIP3 values above $+35 \mathrm{dBm}[16]$, [18], [19].
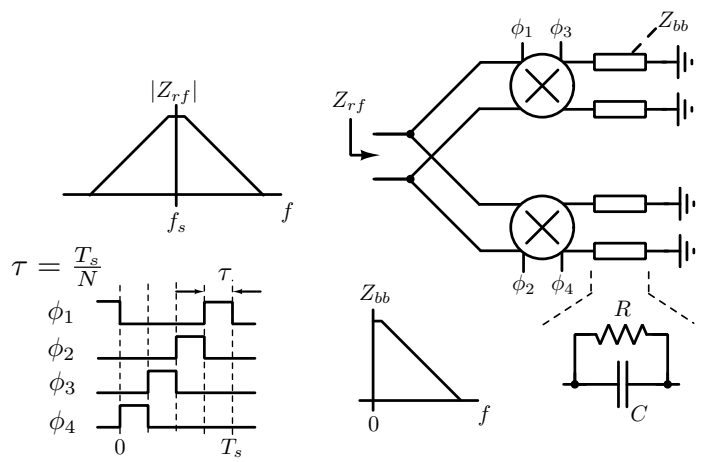

Fig. 1. Upconversion of the baseband impedance $Z_{b b}$ to $f_{s}$ through $N$-path action.

Fig. 2(a) shows a 4-path filter/mixer with 4 capacitors and switches driven by multi-phase non-overlapping digital clocks with frequency $f_{s}$, that defines the center-frequency of the RF filter. Note that, in contrast to Fig. 1, there are no baseband resistors - the signal source resistance $R$ defines the bandwidth instead ${ }^{1}$. The circuit operates as a mixer if the capacitor voltages are used, or an $N$-path filter at the shared RF-node $v_{x}$. Essentially, the lowpass RC filter shape is scaled and shifted to around $f_{s}$. The resulting bandwidth $B W$, defined by the RC time-constant and clock duty cycle, can be as low as a typical communication bandwidth of a few MHz. Since gigahertz clock frequencies (that determine the filters center-frequency) are possible, $Q=f_{s} / B W$ can be very high. As the linearity of passive mixers can be very good, while the properties of switches and capacitors scale favorably with the reduction in the feature size of CMOS technologies, this explains the increased recent interest in $N$-path filters and mixer-first receivers.

The attractive properties of $N$-path filters and mixers bring along design challenges related to linear periodic time variant (LPTV) circuit operation. Time-variance results in signal folding, similar to aliasing in samplers, but with attenuation due to the embedded low-pass filtering [20], [21]. By increasing the number of paths, folding products in a wider signal band can be canceled, but there are practical speed and power limits to multi-phase clock generation. Further, errors in clock phase and capacitor mismatch also limit the achievable cancellation. Hence, assistance by a linear time invariant (LTI) pre-filter, typically a low-pass L-C filter (see

\footnotetext{
${ }^{1} \mathrm{~A}$ resistor is sometimes added to aid impedance matching, but its value is usually significantly higher than $R$, and it plays a secondary role that we neglect.
} 

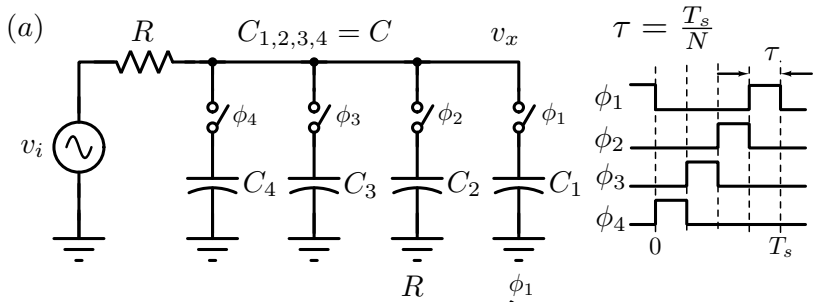

$(b)$
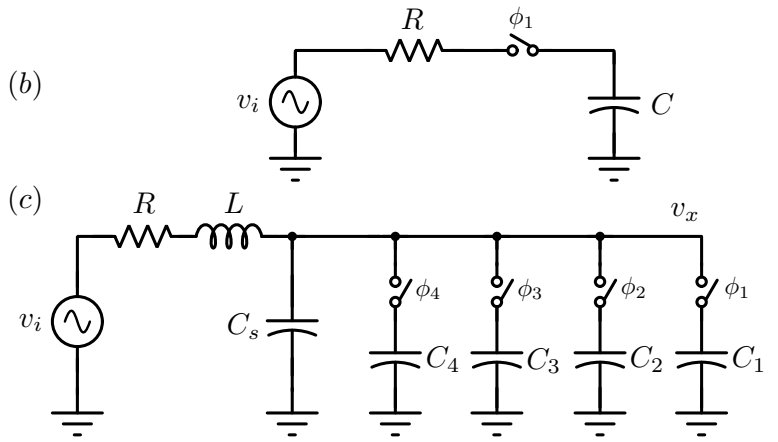

Fig. 2. (a) The switch-RC $N$-path mixer/filter, with $N=4$. (b) The kernel used for analysis. (c) The 4-path mixer/filter with source inductance $L$ and parasitic capacitance $C_{s}$.

Fig. 2(c)), will be needed to achieve sufficient suppression of high-frequency folding. Note that the switched capacitors in Fig. 2(c) now interact with memory elements ( $\mathrm{L}$ and $\mathrm{Cs}$ ) instead of a memoryless resistor. In this paper, we will analyze this interaction and show how it can be exploited to the benefit of $N$-path filter and mixer performance.

For a resistive signal source as in Fig. 2(a), the capacitors do not interact as long as the clocks do not overlap. The circuit can then be split into independent switch-RC kernels [22], as shown in Fig. 2(b). Once the transfer functions and noise of the kernel have been determined, those of the complete network are easily obtained. Several works [21]-[24] have derived the properties of the kernel. A key insight that emerges from these papers is that the voltage sampled on the capacitor plays a crucial role in determining the behavior of the network, not only for discrete-time signal processing, but also when using the time-continuous output of the mixer or $N$-path filter.

If we now add a parasitic capacitance $C_{s}$ at the RF node $v_{x}$, as in Fig. 2(c), baseband capacitors start to interact via $C_{s}$. It turns out that this degrades the gain, noise and selectivity of the $N$-path structure. The kernel approach is no longer applicable, since the filter capacitors $C_{1, \cdots, 4}$ share charge with each other through $C_{s}$. The network now consists of five capacitors and four switches, and the methods of [25] becomes algebraically involved.

It has been observed that adding a series inductor (a nonzero L in Fig. 2(c)) can improve the filter transfer function and reduce noise figure [15], [26], [27]. The inductor, like $C_{s}$, introduces coupling between the four capacitors, again rendering the kernel approach inapplicable. In [26], exact equations for this case were derived for the network of Fig. 2(c) (with $C_{s}=0$ ) using the methods of [25] the resulting frequency-domain analysis is seen to be very lengthy.

In this work, we determine the effect of shunt capacitance and series inductance on the properties of $N$-path mixers and filters. It turns out that, like in the case of Fig. 2(a), the voltages sampled on the capacitors play a crucial role in determining the properties of continuous-time output of the Npath structure. As in [21], we exploit the adjoint network to determine the transfer function of the equivalent LTI filter from the input to sampled capacitor voltages in a simple manner. For a derivation we refer to [21], [28], but the key properties of sampled LPTV networks that we exploit are:

a. The samples of the output of an LPTV system varying at the sampling frequency $f_{s}$, can be thought of as being obtained by sampling the output of an appropriately chosen LTI filter with impulse response denoted by $h_{e q}(t)$.

b. $h_{e q}(t)$ can determined by exciting the adjoint of the original network, as described using Fig. 3.
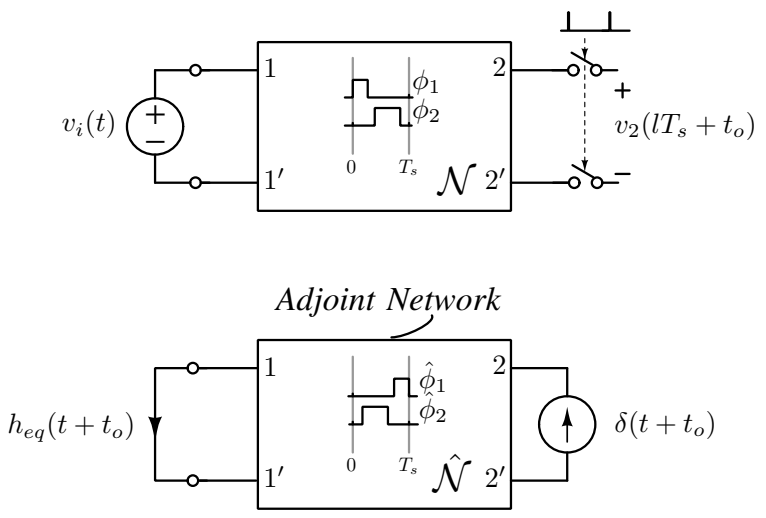

Fig. 3. Determining the impulse response of the equivalent LTI filter corresponding to an LPTV system with sampled outputs using the interreciprocal (or adjoint) network. $l$ is an integer.

When compared to frequency-domain methods, using the adjoint impulse-response method turns out to be simpler, since the impulse "dies" immediately after application (as opposed to analysis with a sinusoidal excitation). We apply this technique to $N$-path mixers and filters with parasitic shunt capacitance and series inductance in the rest of the paper, which is organized as follows. Section II derives the impulse response of the equivalent LTI filter relating the input to the voltage sampled on the capacitors, when the signal source has a parasitic capacitance $C_{s}$. We show that $C_{s}$ not only reduces the peak gain around $f_{s}$, but also causes the peak to shift left. While this phenomenon has been recognized before [20], our analysis quantifies the effect, and gives a fresh timedomain perspective. In Section III, we derive a signal flow graph relating the RF input to the output in the $N$-path filter and passive-mixer modes. Section IV analyzes the case with a series inductor. The intuition gained from Sections II and IV are combined in Section $\mathrm{V}$ to show that an appropriately chosen inductor can largely nullify the deleterious effects of the shunt capacitor, by restoring the gain and reducing the peak-shift of the filtering characteristic. Conclusions are given in Section VI. 


\section{Analysis With the Switch-RC $N$-Path Network With Parasitic Source CAPACITANCE}

The ideal switch-RC $N$-path structure is extended with a parasitic source capacitance $C_{s}$, as shown in Fig. 4, since the switching transistors will introduce parasitic capacitance on the source side.

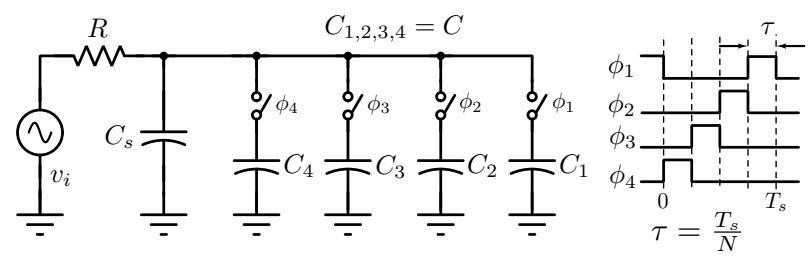

Fig. 4. $N$-path filter/passive-mixer core with a parasitic capacitance $C_{s}$, and switch timing, for $N=4$.

Each of the switches is on for $\tau=T_{s} / N$, and only one switch is on at any given time. Ideal switches (infinite off and zero on resistance) are assumed. The capacitors are named $C_{1, \cdots, 4}$ in the figure to be able to uniquely identify them in the discussion that follows; however, all of them are equal to $C$. The sinusoidal input excitation is denoted by $v_{i}$. The voltage across $C_{1, \cdots, 4}$ are denoted by $v_{1, \cdots, 4}(t)$ respectively. Since the capacitors are equal, it is enough to determine $v_{1}(t)$ - the others can be derived using symmetry. The voltage sampled on $C_{1}$ plays a crucial role in the determination of the transfer functions of the $N$-path structure. Thus, as in our analysis without parasitic capacitance [21], we first determine the equivalent LTI transfer function (whose impulse response is denoted by $h_{e q}(t)$ ) that relates $v_{i}$ to $v_{1}(t)$ at the falling edges of $\phi_{1}$ i.e, $v_{1}\left[k T_{s}\right]$. As seen in Fig. $4, C_{s}$ introduces coupling between the $N$ capacitors, and the "independent kernel" approach of [22] cannot be used. Without loss of generality, we analyze the circuit for $N=4$. The results are readily extended to arbitrary $N$.

To determine $h_{e q}(t)$, we form the adjoint network, as shown in Fig. 5(a). Note that all the clock signals, denoted by the hatted symbols, are time-reversed in the adjoint. The voltages across the capacitors $C_{1, \cdots, 4}$ are denoted by $\hat{v}_{1, \cdots, 4}$ respectively. $\hat{v}_{x}$ denotes the voltage across $C_{s}{ }^{2}$ Next, we inject a current impulse into $C_{1}$ at the rising edge of $\hat{\phi}_{1}$. From the theory of the adjoint network, the current waveform through the resistor is the desired $h_{e q}(t)$ [28]. Upon application of the impulse, $C_{1}$ and $C_{s}$ are instantly charged to $\hat{v}_{1}(0+)=1 /\left(C+C_{s}\right)$. Thus, $h_{e q}(t)$ is the current waveform in $R$ that results when $C_{1}$ and $C_{s}$ are charged to $1 /\left(C+C_{s}\right)$ at $t=0+$.

When $\hat{\phi}_{1}$ is high, $\hat{v}_{1}$ and $\hat{v}_{x}$ are identical, and decay exponentially with a time-constant $R\left(C+C_{s}\right)$. At $t=\tau-$, $\hat{v}_{1}(\tau-)=\hat{v}_{1}(0+) e^{-\tau / R\left(C+C_{s}\right)} \equiv \beta \hat{v}_{1}(0+)$ (see the table below Fig. 5(a)). If the voltage across the relevant capacitor at the beginning of the "on" phase of the switch is known, $\hat{v}_{x}$ during the rest of the phase can be determined, since the initial voltage decays exponentially with time-constant $R\left(C+C_{s}\right)$. To determine $\hat{v}_{x}(t)$ (which equals $h_{e q}(t) / R$ ), therefore, it

\footnotetext{
${ }^{2}$ The hats remind us that we are dealing with quantities pertaining to the adjoint network.
}

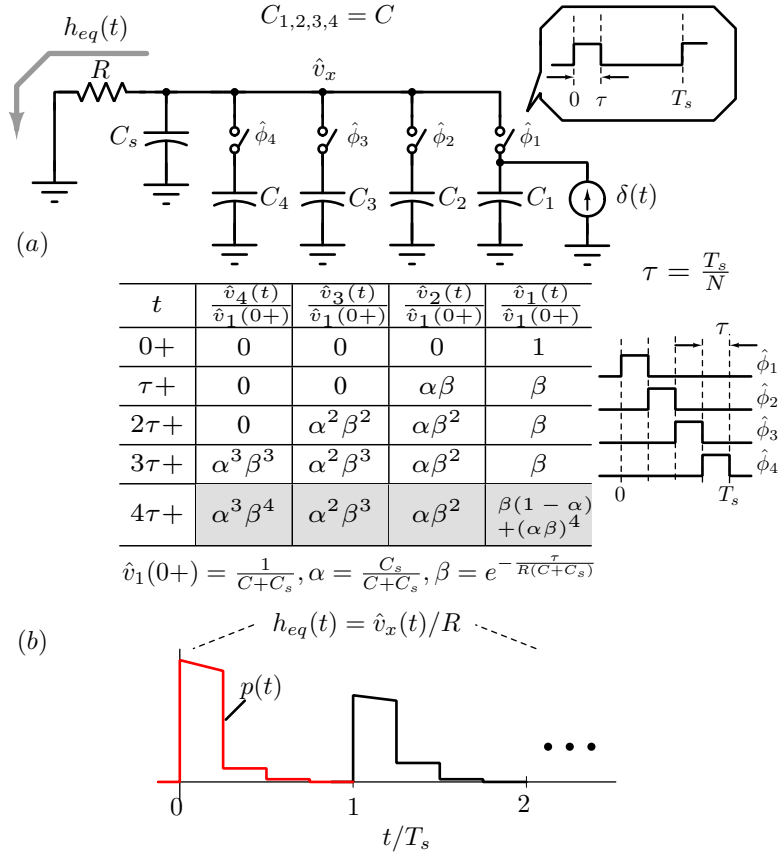

Fig. 5. (a) The adjoint network, with voltages on the capacitors indicated at the beginning of the first and second clock cycles. (b) A representative $\hat{v}_{x}$ waveform; the portion for $0 \leq t<T_{s}$ is denoted by $p(t)$. For practical values of $C_{s}, C$ and $N, \hat{v}_{x} \approx 0$ at $t=T_{s}-$. Note that $h_{e q}(t)=\hat{v}_{x} / R$.

suffices to keep track of $\hat{v}_{1, \cdots, 4}$ at the beginning of the "on" phase of every switch signal. Further, by exploiting symmetry and linearity, only the samples of $\hat{v}_{1, \cdots, 4}$ at $4 \tau+$ can be used to determine $h_{e q}(t)$ for all time, as shown later in this section. We now proceed to determine $\hat{v}_{1, \cdots, 4}(4 \tau+)$.

At $t=\tau-, C_{1}$ is disconnected from the network and $C_{2}$, which was initially discharged, is connected to $C_{s}$. The voltage across $C_{1}$ is thus held at $\beta \hat{v}_{1}(0+)$ for the remainder of the clock cycle. Due to charge sharing, the voltage across $C_{2}$ at $t=\tau+$ is

$$
\hat{v}_{2}(\tau+)=\underbrace{\beta \hat{v}_{1}(0+)}_{\hat{v}_{1}(\tau-)} \underbrace{\frac{C_{s}}{\left(C+C_{s}\right)}}_{\alpha}=\alpha \beta \hat{v}_{1}(0+)
$$

where $\alpha=C_{s} /\left(C+C_{s}\right)$ and $\beta=e^{-\tau / R\left(C+C_{s}\right)}$. In a similar fashion, exponential decay and charge sharing with $C_{3}$ and $C_{4}$ occur when $\hat{\phi}_{3}$ and $\hat{\phi}_{4}$ are high respectively, and we have

$$
\begin{aligned}
& \hat{v}_{3}(2 \tau+)=\hat{v}_{x}(2 \tau+)=(\alpha \beta)^{2} \hat{v}_{1}(0+) \\
& \hat{v}_{4}(3 \tau+)=\hat{v}_{x}(3 \tau+)=(\alpha \beta)^{3} \hat{v}_{1}(0+) .
\end{aligned}
$$

The table in Fig. 5(a) shows $\hat{v}_{1, \cdots, 4}$ normalized to $\hat{v}_{1}(0+)$, at the beginning of integer multiples of $\tau+$. Let us now examine the voltages across the capacitors at $t=$ $4 \tau+=T_{s}+$. When $\hat{\phi}_{1}$ goes high at $4 \tau+$, the voltage across $C_{1}$, which was $\beta \hat{v}_{1}$, is now reduced by a factor $C /\left(C+C_{s}\right)=(1-\alpha)$ due to charge sharing with $C_{s}$, which had a voltage $\beta \hat{v}_{4}(3 \tau+)=\alpha^{3} \beta^{4} \hat{v}_{1}(0+)$ at $t=4 \tau-$. Thus, at $t=T_{s}+$,

$$
\begin{aligned}
\hat{v}_{1}(4 \tau+) & =\frac{C}{C+C_{s}} \hat{v}_{1}(\tau-)+\frac{C_{s}}{C+C_{s}} \hat{v}_{4}(4 \tau-) \\
& =\left(\beta(1-\alpha)+\alpha^{4} \beta^{4}\right) \hat{v}_{1}(0+) .
\end{aligned}
$$


This result makes intuitive sense as the first term relates to charge stored on $C_{1}(=C)$ which is shared with $C_{s}$, while the second relates to charge on $C_{s}$ shared with $C_{1}$.

Having determined the voltages on the capacitors at $t=4 \tau+=T_{s}+$, we are now in a position to determine $h_{e q}(t)$. Recall that $h_{e q}(t)$ is the current through the resistor due to an initial voltage of $\hat{v}_{1}(0+)$ on $C_{1}$ and $C_{s}$, with all other capacitor voltages being zero. Let us denote the waveform $h_{e q}(t)$ for $0 \leq t<T_{s}$ by $p(t)$, as shown in red in Fig. 5(b). The voltages across $C_{1, \cdots, 4}$ at $t=4 \tau+=T_{s}+$ are shown in grey in the table of Fig. 5(a). Their contributions to $h_{e q}(t)$ can be evaluated using superposition by considering them one at a time (i.e., all other capacitor voltages being zero). At $t=4 \tau+=T_{s}+, C_{1}$ and $C_{s}$ have a voltage $\left(\beta(1-\alpha)+(\alpha \beta)^{4}\right) \hat{v}_{1}(0+)$. Due to linearity and the LPTV nature of the network, the contribution of this voltage to $h_{e q}(t)$ can be written as $\left(\beta(1-\alpha)+(\alpha \beta)^{4}\right) h_{e q}\left(t-T_{s}\right)$. In a similar fashion, using symmetry, the contributions of the voltages across $C_{2,3,4}$ can be expressed in terms of $h_{e q}(t)$, with appropriate scaling factors and delays. Hence, $h_{e q}(t)$ consists of five contributions: $p(t)$, that describes $h_{e q}(t)$ in the initial clock cycle, followed by contributions from each of the four capacitors. $h_{e q}(t)$ can thus be recursively written as follows.

$$
\begin{gathered}
h_{e q}(t)=\underbrace{p(t)}_{h_{e q}(t) \text { for } 0 \leq t<T_{s}} \quad+ \\
\underbrace{\left(\beta(1-\alpha)+(\alpha \beta)^{4}\right) h_{e q}\left(t-T_{s}\right)}_{h_{e q}(t) \text { for } t>T_{s} \text { due to } \hat{v}_{1}(4 \tau+)}+\underbrace{\alpha(1-\alpha) \beta^{2} h_{e q}\left(t-T_{s}-\tau\right)}_{h_{e q}(t) \text { for } t>T_{s} \text { due to charge on } C_{2}} \\
+\underbrace{\alpha^{2}(1-\alpha) \beta^{3} h_{e q}\left(t-T_{s}-2 \tau\right)}_{h_{e q}(t) \text { for } t>T_{s} \text { due to charge on } C_{3}}+\underbrace{\alpha^{3}(1-\alpha) \beta^{4} h_{e q}\left(t-T_{s}-3 \tau\right)}_{h_{e q}(t) \text { for } t>T_{s} \text { due to charge on } C_{4}} .
\end{gathered}
$$

Note that the $(1-\alpha)$ terms appear due to attenuation when the capacitors share charge with $C_{s}$. Applying the Fourier transform to both sides of the equation above, we have

$$
H_{e q}(f)=\frac{P(f)}{\left[\begin{array}{r}
1-\left(\beta(1-\alpha)+(\alpha \beta)^{4}\right) z^{-4}-\alpha(1-\alpha) \beta^{2} z^{-5} \\
-\alpha^{2}(1-\alpha) \beta^{3} z^{-6}-\alpha^{3}(1-\alpha) \beta^{4} z^{-7}
\end{array}\right]} .
$$

where

- $P(f)$ is the Fourier transform of $h_{e q}(t)$ for $0 \leq t<T_{s}$.

- $\alpha=C_{s} /\left(C_{s}+C\right), \beta=\exp \left(-\tau /\left(R\left(C+C_{s}\right)\right)\right)$ and

- $z=\exp (j 2 \pi f \tau)$.

Note that $H_{e q}(f)$ is the transfer function of a continuous-time filter, and $z$ is short hand for $\exp (j 2 \pi f \tau)$. The astute reader might wonder why $z=e^{j 2 \pi f \tau}$ is used (as opposed to $e^{j 2 \pi f T_{s}}$ ). There are two reasons for this choice.

a. Though the clock period is $T_{s}$, the input is sampled on to one of the capacitors every $\tau$ seconds, making the effective sampling rate $1 / \tau$. Thus, using $z=e^{j 2 \pi f \tau}$ makes sense.

b. On the practical front, choosing $z=e^{j 2 \pi f \tau}$ results in integer powers of $z$ in the expressions for $H_{e q}(f)$. If $z$ was chosen to be $z=e^{j 2 \pi f T_{s}}$ the fractional powers of $z$ that would appear in our expressions could lead to computation errors unless great care is exercised. Integer powers of $z$ avoid this problem.
To determine $P(f)$, we note that $p(t)$ for $0 \leq t<\tau$ is an exponentially decaying pulse given by

$$
p(t)=\frac{1}{R\left(C+C_{s}\right)} e^{-\frac{t}{R\left(C+C_{s}\right)}} u(t), 0 \leq t<\tau .
$$

where $u(t)$ is the unit-step function. The Fourier transform of this part of $p(t)$ is given by $\left(1-\beta e^{-j 2 \pi f \tau}\right) /\left(1+j 2 \pi f R\left(C+C_{s}\right)\right)$. For $\tau \leq t<2 \tau$, the shape of $p(t)$ is the same, but has a peak value of $\alpha \beta \hat{v}_{1}(0+)$. Reasoning similarly for $2 \tau \leq t<3 \tau$ and $3 \tau \leq t<4 \tau$, and using the table in Fig. 5(a), we have

$$
\begin{aligned}
P(f) & =\frac{\left(1-\beta e^{-j 2 \pi f \tau}\right)\left(1+\alpha \beta z^{-1}+(\alpha \beta)^{2} z^{-2}\right.}{\left.+(\alpha \beta)^{3} z^{-3}\right)} \\
& =\frac{\left(1-\beta e^{-j 2 \pi f \tau}\right)}{1+j 2 \pi f R\left(C+C_{s}\right)} \frac{1-\left(\alpha \beta z^{-1}\right)^{4}}{1-\alpha \beta z^{-1}}
\end{aligned}
$$

(4), along with (6), yields $H_{e q}(f)$ for arbitrary $C_{s}$.

As a sanity check, when $C_{s}=0$ and $\alpha=0$,

$$
H_{e q}(f)=\frac{1}{1+j 2 \pi f R C} \frac{1-\beta e^{-j \pi f \tau}}{1-\beta e^{-j 2 \pi f T_{s}}}
$$

where $\beta=\exp (-\tau / R C)$. This is consistent with the results of [21]-[23].

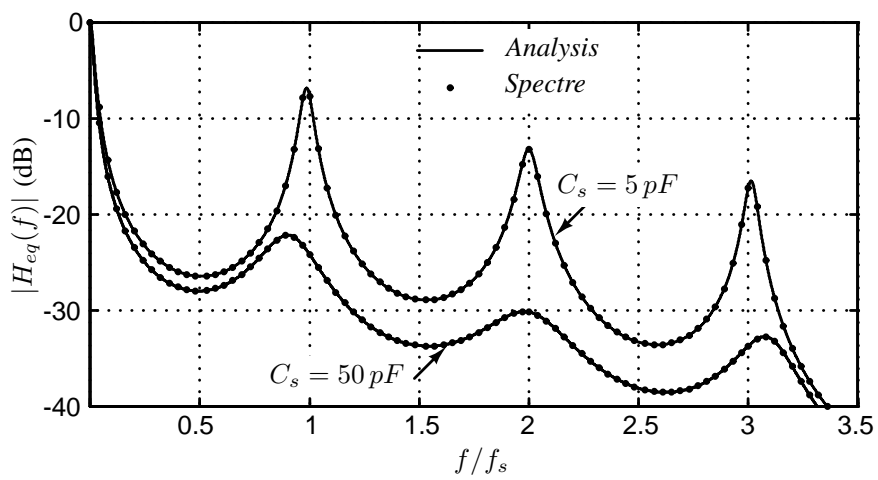

Fig. 6. Comparison of analytical and simulated $\left|H_{e q}(f)\right|$, for $C_{s}=5 \mathrm{pF}$ $(\alpha=0.1)$ and $C_{s}=50 \mathrm{pF}(\alpha=1) . N=4, C=50 \mathrm{pF}, \mathrm{R}=50 \Omega$ and $f_{s}=1 \mathrm{GHz}$.

Fig. 6 compares the analytically determined $\left|H_{e q}(f)\right|$ with that obtained from simulations ${ }^{3}$, for $\alpha=0.1$ and $\alpha=1$. Excellent agreement is seen. For $\alpha=1, C_{s}=C$, a situation that will probably not occur in practice; the purpose of illustration is to demonstrate the accuracy of our analysis even for very large $C_{s}$.

As mentioned in the introduction, and seen in [21], [23], the sampled capacitor voltage plays a crucial role in the performance of the switched-RC circuit. The presence of $C_{s}$ does not alter this fact, as we will show in Section III. Anticipating this, we now examine the behaviour of $H_{e q}(f)$. It turns out that this gives valuable insights into the operation of the network as a passive mixer or $N$-path filter.

Fig. 7(a) shows $\left|H_{e q}(f)\right|$ for different values of $C_{s}$ (where $\alpha$ ranges from $0-0.2$ ), for $N=4, C=50 \mathrm{pF}, R=50 \Omega$ and

\footnotetext{
${ }^{3} H_{e q}(f)$ can be obtained from simulations using the sampled-PXF analysis.
} 

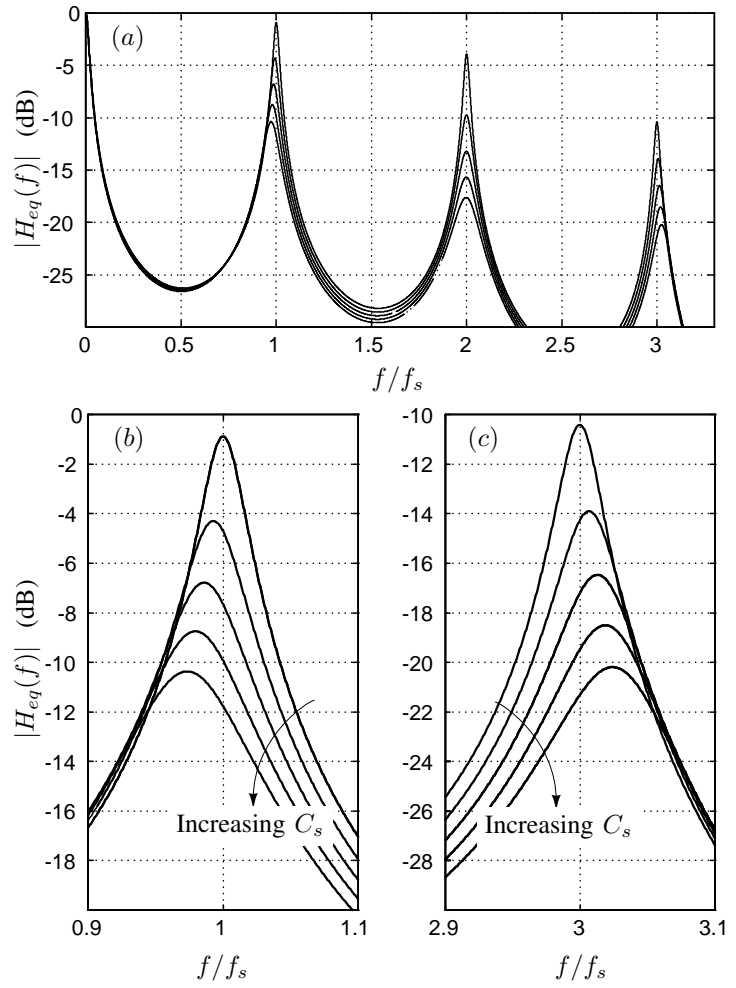

Fig. 7. $\left|H_{e q}(f)\right|$ for $C_{s}=0,2.5,5,7.5$ and $10 \mathrm{pF}$. $N=4, C=50 \mathrm{pF}$, $\mathrm{R}=50 \Omega$ and $f_{s}=1 \mathrm{GHz}$

$f_{s}=1$ GHz. Figs. 7(b) and (c) are zooms of $\left|H_{e q}(f)\right|$ around $f=f_{s}$ and $f=3 f_{s}$ respectively. We see that increasing $C_{s}$ reduces the gain of $\left|H_{e q}(f)\right|$ at $f_{s}$, but also results in a less selective response. We see that $C_{s}$ causes the peak of the responses to shift. Interestingly, for frequencies around $f_{s}$, the peaks shift left, for those around $2 f_{s}$, the peaks do not shift, while they shift right for $f \approx 3 f_{s}$. We now give more intuition for each of these effects.

In a well-designed practical $N$-path structure, $\alpha$ will be small (typically less than 0.1 ) to avoid the reduction in gain and selectivity. (4) and (6) can then be approximated by neglecting terms containing $\alpha^{2}$ and higher powers of $\alpha$. We then have

$$
\begin{aligned}
H_{e q}(f) & \approx \frac{P(f)}{1-\beta(1-\alpha) z^{-4}-\alpha(1-\alpha) \beta^{2} z^{-5}} \\
P(f) & \approx \frac{\left(1-\beta e^{-j 2 \pi f \tau}\right)}{\left(1+j 2 \pi f R\left(C+C_{s}\right)\right)\left(1-\alpha \beta z^{-1}\right)} .
\end{aligned}
$$

The equations above have a form similar to that when $C_{s}=0$ (where $\alpha=0$ ), except for a $z^{-5}$ and $z^{-1}$ terms in the denominators of $H_{e q}(f)$ and $P(f)$ respectively. Looking back at our derivation, we see that these terms are due to the coupling between $C_{1}$ and $C_{2}$, introduced by $C_{s}$. From these equations, therefore, it is seen that for all practical purposes, the coupling between $C_{1}$ and $C_{3,4}$ are not important; it is the interaction between $C_{1}$ and $C_{2}$ (which is the capacitor that is switched in next) that matters.

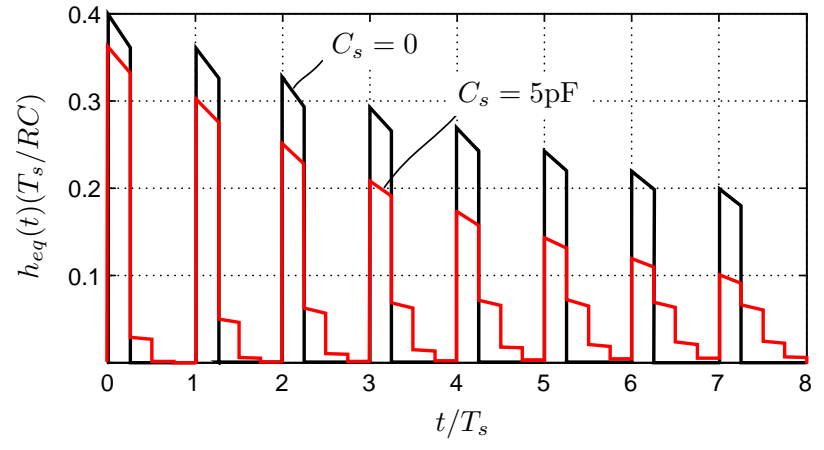

Fig. 8. $h_{e q}(t)$ for $C_{s}=0$ and $C_{s}=5 \mathrm{pF} . N=4, C=50 \mathrm{pF}, \mathrm{R}=50 \Omega$ and $f_{s}=1 \mathrm{GHz}$.

\section{A. Gain and $Q$ Reduction}

Fig. 8 shows the impulse responses of the equivalent LTI filters relating $v_{i}$ to the voltage sampled on $C_{1}$, for $\alpha=0$ and $\alpha=0.1$. We notice two differences with a non-zero $C_{s}$. First the peak value is reduced with respect to when $C_{s}=0$. Next, the response decays much faster - as anticipated from the smaller coefficient of the $z^{-4}$ term in the denominator of (8). We skip the details here, but calculations show that for small $\alpha$, the reduction in the peak gain due to a non-zero $C_{s}$, for $f \approx f_{s}$ is approximately $(1-\beta) /(1-\beta(1-\alpha))$.

\section{B. Peak Shift}

The shift in the peaks of the transfer function around $f=f_{s}$ can be understood by evaluating the denominator of $H_{e q}(f)$ in (8) for $z=e^{j 2 \pi T_{s}\left(f_{s}+\Delta f\right) / 4}$.

$$
H_{e q}\left(f_{s}+\Delta f\right)=\frac{P\left(f_{s}+\Delta f\right)}{1-\beta(1-\alpha) e^{-j 2 \pi \Delta f T_{s}}} .
$$

Since $\Delta f \ll f_{s}$ and $\alpha \ll 1$, using $e^{-j 2 \pi \Delta f T_{s}} \approx 1-j 2 \pi \Delta f T_{s}$ and $e^{-j 2.5 \pi \Delta f T_{s}} \approx 1$, this can be approximated as

$H_{e q}\left(f_{s}+\Delta f\right) \approx \frac{P\left(f_{s}\right)}{1-\beta(1-\alpha)+j \beta(1-\alpha)\left(2 \pi \Delta f T_{s}+\alpha \beta\right)}$.

The magnitude of $H_{e q}$ will attain its maximum at that frequency where the magnitude of its denominator is minimum. This occurs when the last term of the denominator becomes zero, which corresponds to

$$
\frac{\Delta f}{f_{s}}=-\frac{\alpha \beta}{2 \pi} .
$$

Thus, the peak in the response occurs at a frequency less than $f_{s}$. For the response around $3 f_{s}, H_{e q}(f)$ can be approximated as

$$
H_{e q}\left(3 f_{s}+\Delta f\right) \approx \frac{P\left(3 f_{s}\right)}{1-\beta(1-\alpha)+j \beta(1-\alpha)\left(2 \pi \Delta f T_{s}-\alpha \beta\right)} .
$$

Thus, around $3 f_{s}$, the peak is shifted to the right by $f_{s} \alpha \beta /(2 \pi)$. Similar analysis leads us to conclude that there should be no peak shift for frequencies around $2 f_{s}$. The analytical and simulated peak frequency deviations around $f_{s}$, for varying $C_{s}$, are shown in Fig. 9. As seen in the analysis 


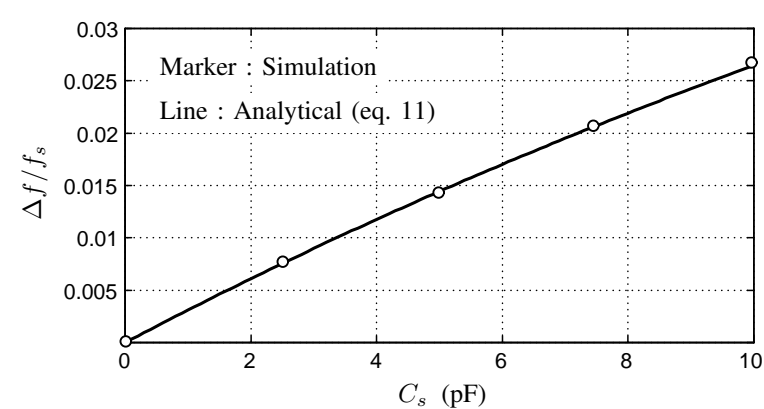

Fig. 9. Deviation of the peak frequency from $f_{s}$ as a function of $C_{s} . C=$ $50 \mathrm{pF}, R=50 \Omega, f_{s}=1 \mathrm{GHz}$ and $N=4$.

above, peak-frequency shift is due to the $z^{-5}$ term in the denominator of $H_{e q}(f)$, the root cause of which is the coupling between $C_{1}$ and $C_{2}$ through $C_{s}$.

\section{Complete Output WaVEForm: $N$-PATH AND PASSIVE-MIXER MODE OF OPERATION}

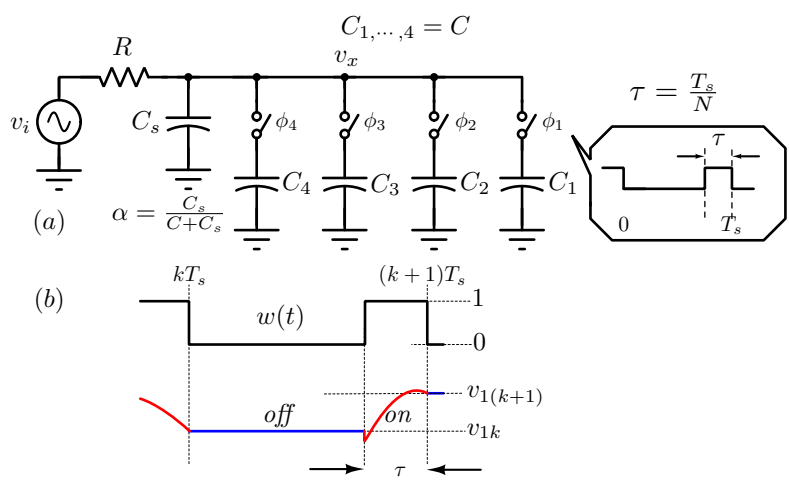

(c)

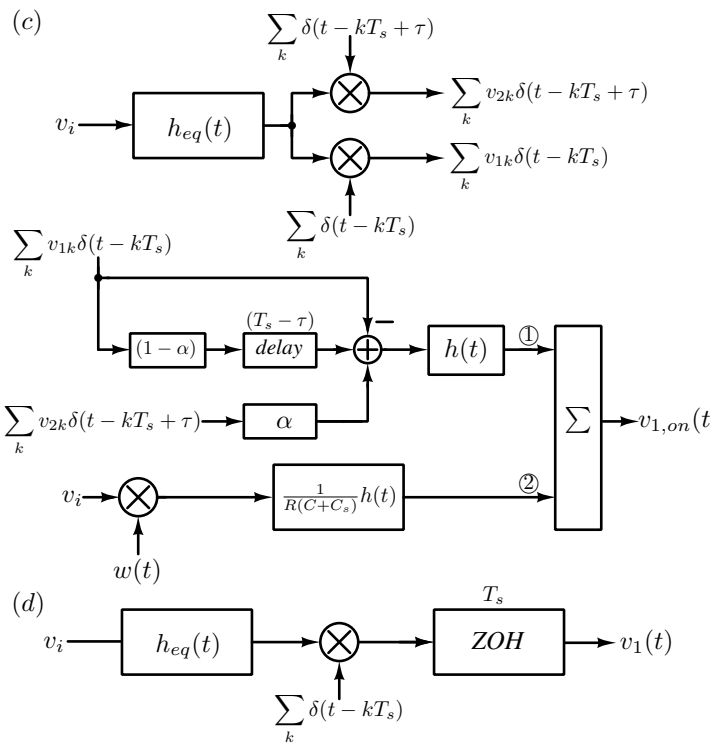

Fig. 10. (a) $N$-path circuit with parasitic capacitance, driven by a sinusoidal input $v_{i}(t)$. (b) $v_{1}(t)$ and $w(t)$ as defined in [21]. (c) Signal-flow graph relating $v_{1, o n}(t)$ to $v_{i}$, needed for $N$-path operation. (d) Approximation to $v_{1}(t)$ in the passive-mixer mode of operation.

So far, we determined the impulse response $\left(h_{e q}(t)\right)$ of the equivalent LTI filter that would produce the same samples as $v_{1}(t)$ (sampled at the falling edge of $\phi_{1}$ ) in the $N$-path structure of Fig. 4. This is all that would be needed if we processed the samples, as in a switched-capacitor filter or A/D converter. The output of an $N$-path filter or a mixer-first switch-RC receiver (with implicit $N$-path filtering), however, is often used in a time-continuous way. In such circuits, therefore, it is necessary to know the entire output voltage across the capacitors. Fortunately, it is adequate to determine the voltage across one of the capacitors; the others can be found by symmetry. In the discussion that follows, therefore, we focus on $v_{1}(t)$, which the voltage on $C_{1}$ in response to an excitation $v_{i}(t)$ (see Fig. 10(a)).

$v_{1}(t)$ is seen to be comprised of two waveforms, $v_{1, o n}(t)$ that is non-zero when $\phi_{1}$ is high, and $v_{1, \text { off }}(t)$, which is non-zero when $\phi_{1}$ is low, as shown in red and blue in Fig. 10(b) respectively. $v_{1, o n}(t)$ is of interest when the circuit of Fig. 10(a) is operated as an $N$-path filter, since the output is $v_{x}$, which is the sum of the 'on' portions of the waveforms $v_{1, \cdots, 4}(t) \cdot v_{1, \text { off }}(t)$ for $k T_{s}<t<(k+1) T_{s}-\tau$ is simply a zero-order-hold (ZOH) version of $v_{1}\left[k T_{s}\right] \equiv v_{1 k}$.

The signal-flow graph for the $v_{1}(t)$ can be developed as described in [21], with additional branches needed to account for charge-sharing. The details are omitted here due to space constraints. It turns out that the flow-graph reduces that of Fig. 22 in [21] when $\alpha=0$. We discuss below the relevant aspects of the the signal-flow graph during the $N$-path filter and passive-mixer modes of operation.

\section{A. Operation as an N-Path Filter}

The signal-flow graph that relates $v_{i n}(t)$ to $v_{1, \text { on }}(t)$ is shown in Fig. 10(c), where $h(t)=e^{-t /\left(R\left(C+C_{s}\right)\right)}$. Since the voltages across $C_{1}$ and $C_{2}$ are coupled through $C_{s}$, it is necessary to keep track of the samples of $v_{2}$, denoted by $v_{2 k}$. A non-zero $\alpha$ has the following effects:

a. extra loss in path (1), as charge sharing with $C_{s}$ result is extra loss, and is modeled by the factor $(1-\alpha)$.

b. $v_{2 k}$ contributes to the output through the factor $\alpha$ due to charge-sharing.

c. the time-constant of the exponential decay is $R\left(C+C_{s}\right)$ instead of $R C$.

When $v_{i}=e^{j 2 \pi f t}$, the component of $v_{1, o n}(t)$ at the frequency $f$, is the sum of contributions from paths (1) and (2), which are seen to be

$$
-\underbrace{\frac{H_{e q}(f)}{T_{s}}}_{\substack{\mathcal{F}\left(h_{e q}(t)\right) \\ \text { and sampling }}} \underbrace{\frac{R\left(C+C_{s}\right)}{1+j 2 \pi f R\left(C+C_{s}\right)}}_{\mathcal{F}(h(t))}\left(1-e^{-j 2 \pi f\left(T_{s}-\tau\right)}\right)(1-\alpha)
$$$$
\text { Path (2) } \rightarrow \underbrace{\frac{\tau}{T_{s}}}_{\begin{array}{c}
\text { dc component } \\
\text { of } w(t)
\end{array}} \cdot \underbrace{\frac{1}{1+j 2 \pi f R\left(C+C_{s}\right)}}_{\mathcal{F}\left(h(t) / R\left(C+C_{s}\right)\right)} \text {. }
$$ 
Thus, $X_{0}(f)$, the zeroth-order harmonic transfer function of the $N$-path filter, given by $N \cdot V_{1, o n}(f) / e^{j 2 \pi f t}$, and using $N \tau=T_{s}$, is seen to be

$$
\begin{gathered}
X_{0}(f)=\frac{1}{\left(1+j 2 \pi f R\left(C+C_{s}\right)\right)}[1- \\
\left.\frac{R\left(C+C_{s}\right)}{\tau} H_{e q}(f)(1-\alpha)\left\{1-e^{-j 2 \pi f\left(T_{s}-\tau\right)}\right\}\right] .
\end{gathered}
$$

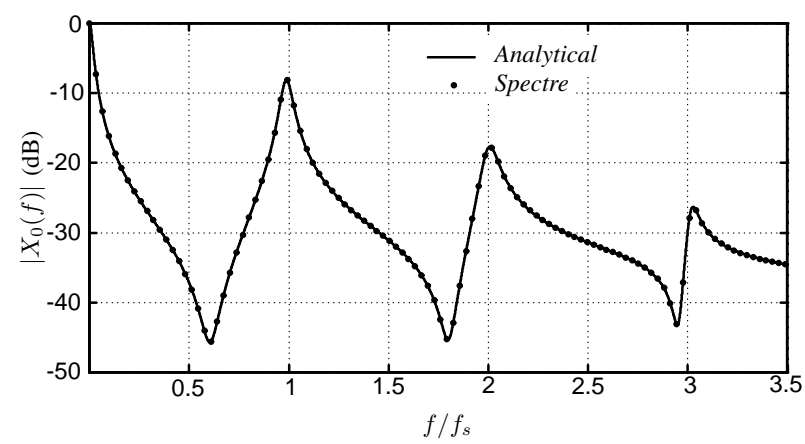

Fig. 11. Analytical versus spectre PAC simulations in the $N$-path filter mode. $R=50 \Omega, C=50 \mathrm{pF}, C_{s}=5 \mathrm{pF}, f_{s}=1 \mathrm{GHz}$.

Fig. 11 compares $\left|X_{0}(f)\right|$ determined analytically with that obtained from a periodic-AC simulation, for $f_{s}=1 \mathrm{GHz}$, $C=50 \mathrm{pF}, R=50 \Omega$ and $\alpha=1 / 11$. Excellent agreement is seen.

Higher order harmonic transfer functions can similarly be determined from the signal-flow graph of Fig. 10(d). Space constraints prevent us from going into a detailed discussion here, but the conclusion is that higher-order harmonic transfer functions are (also) largely determined by the behavior of $H_{e q}(f)$.

\section{B. Passive-Mixer Mode of Operation}

When operated as a passive mixer, the complete voltage waveforms across the capacitors are relevant. The input frequency is very high compared to the bandwidth of the $R C$ network. Further, $R C \gg \tau$, which means that $\beta$ is close to unity. As we have already discussed, $v_{1, \text { off }}(t)$ is simply $v_{1 k}$ held for a duration of $\left(T_{s}-\tau\right)$. When $\phi_{1}$ goes high, $C_{1}$ shares charge with $C_{s}$, attenuating its voltage by a factor $(1-\alpha)$. If $\alpha$ is small, this can be neglected. Further, since $R C \gg \tau$, $v_{1, \text { on }}(t)$ can be approximated to $v_{1 k}$ held for a duration $\tau$. Thus, as in the case with $C_{s}=0$, the output in the passive mixer mode can be thought of as the sampled version of the voltage across $C_{1}$, held for a whole clock period. The signalflow graph relating $v_{i}(t)$ to $v_{1}(t)$ in the passive-mixer mode can therefore be approximated as shown in Fig. 10(d).

Fig. 12 compares the results of our analysis with periodic transfer function (PXF) simulations from Spectre. Since the PXF analysis considers the entire waveform across the capacitor, the good agreement with our approximate analysis confirms that the sampled output of the capacitor is almost all that matters in the mixer region, as far as the loss and shape around the peaks is concerned. $\left|H_{e q}\right|$ when $C_{s}=0$ is also shown for comparison - we see significant drop in the gain

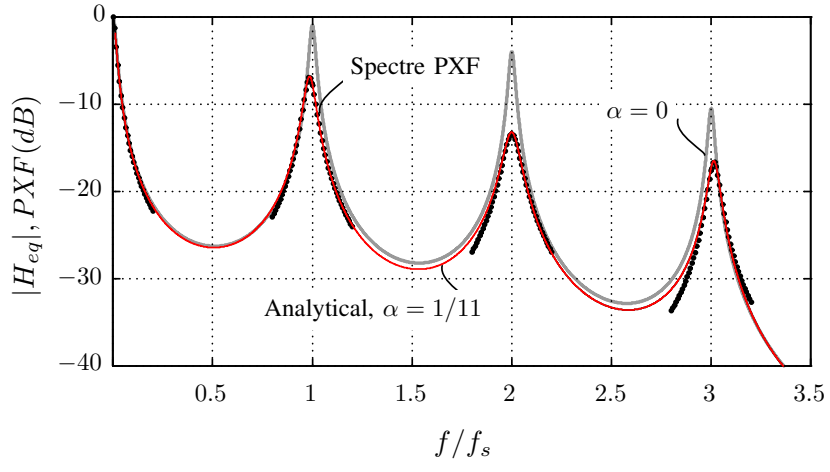

Fig. 12. Analytical versus spectre PXF simulations: for the passive-mixer mode, with $R=50 \Omega, C=50 \mathrm{pF}, C_{s}=5 \mathrm{pF}(\alpha=1 / 11), f_{s}=1 \mathrm{GHz}$. The response for $C_{s}=0$ is also shown for reference.

at $f_{s}$. From the analysis in Section II.B, this gain should drop by about $(1-\beta) /(1-\beta(1-\alpha))=5.8 \mathrm{~dB}$, as confirmed from Fig. 12.

Far-out maximum filter attenuation is not accurately predicted by the signal-flow graph of Fig. 10(d), but this is often limited in practice by other effects that are not modeled here, e.g. switch resistance. The aim of this work is the firstorder modeling of the key filter parameters: insertion-loss and close-in filter roll-off.

\section{ANALysis With Source Inductance}

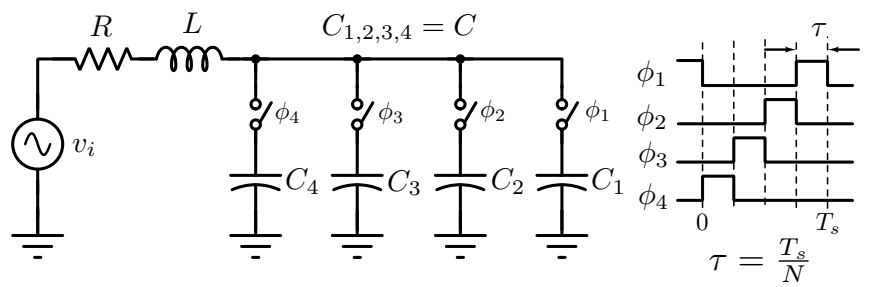

Fig. 13. $N$-path switching mixer with an inductive source impedance, with $N=4$.

In this section, we analyze the $N$-path passive mixer with an inductive source impedance, as shown in Fig. 13. We assume instantaneous switching as in [26], so that the inductor immediately connects from one capacitor to another. Like the case with the parasitic capacitor, the inductor introduces coupling between the four paths, complicating analysis. Further, we assume the practical situation, where $R C \gg L / R$. To find the impulse response of the equivalent LTI filter relating $v_{i}$ to the sampled voltage across $C_{1}$, we form the adjoint network as shown in Fig. 14(a). We excite $C_{1}$ with an impulsive current at $t=0$, resulting in a voltage $\hat{v}_{x}(0+)=1 / C$. Thanks to the inductor, the current through the resistor is smaller than what it would have otherwise been, as shown in Fig. 14(b). Thus, after a time $\tau$, the capacitor voltage is larger than what it would have been, had $L$ been 0 . Intuitively, therefore, we must expect the waveforms to "last" for a much larger time when compared to the case without the inductor. This means that the $Q$ of the resulting filter should be higher. When the inductor is switched to $C_{2}$ (which was initially uncharged), the $\hat{i}_{L}(t)$, 


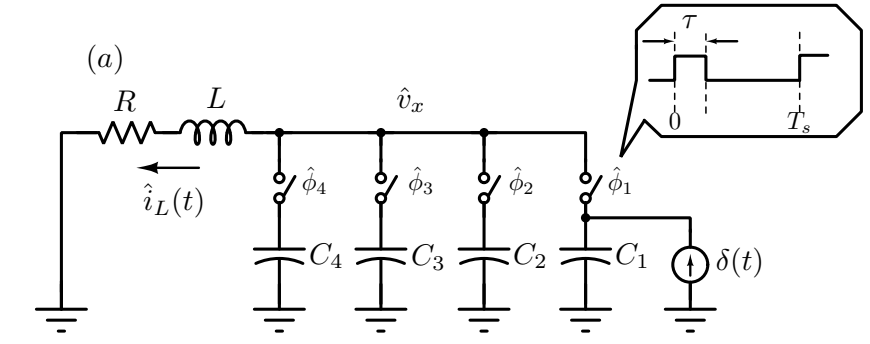

\begin{tabular}{c|c|c|c|c|c}
\hline$t$ & $\hat{i}_{L}(t)$ & $-\frac{\hat{v}_{4}(t)}{\hat{v}_{1}(0+)}$ & $-\frac{\hat{v}_{3}(t)}{\hat{v}_{1}(0+)}$ & $-\frac{\hat{v}_{2}(t)}{\hat{v}_{1}(0+)}$ & $\frac{\hat{v}_{1}(t)}{\hat{v}_{1}(0+)}$ \\
\hline $0+$ & 0 & 0 & 0 & 0 & 1 \\
\hline$\tau+$ & $h_{L}(\tau)$ & 0 & 0 & 0 & $\beta$ \\
\hline $2 \tau+$ & $\alpha h_{L}(\tau)$ & 0 & 0 & $L C h_{L}^{2}(\tau)$ & $\beta$ \\
\hline $3 \tau+$ & $\alpha^{2} h_{L}(\tau)$ & 0 & $\alpha L C h_{L}^{2}(\tau)$ & $L C h_{L}^{2}(\tau)$ & $\beta$ \\
\hline $4 \tau+$ & $\alpha^{3} h_{L}(\tau)$ & $\alpha^{2} L C h_{L}^{2}(\tau)$ & $\alpha L C h_{L}^{2}(\tau)$ & $L C h_{L}^{2}(\tau)$ & $\beta$ \\
\hline
\end{tabular}

(b)
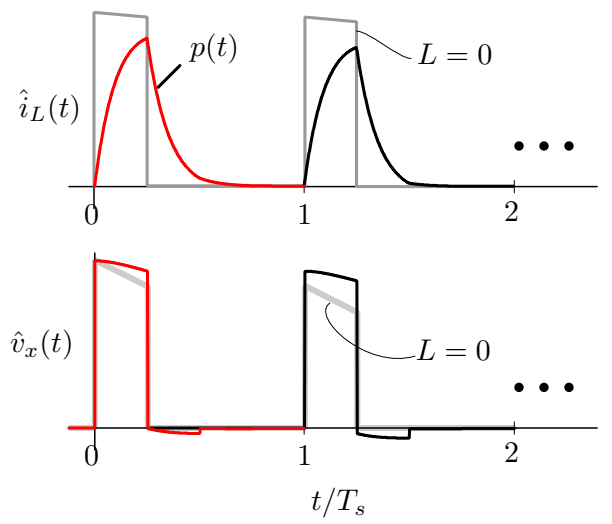

Fig. 14. (a) The adjoint network. (b) Representative inductor current and $\hat{v}_{x}$ waveforms. The corresponding waveforms when $L=0$ are shown in grey.

which was established in the previous clock phase, continues to flow in the same direction, thereby causing $\hat{v}_{x}$ to become negative in the second phase, as shown in Fig. 14(b). This trend continues in the third and fourth intervals. For practical values of $L$, the inductor is virtually "disfluxed" at $t=4 \tau-=T_{s}-$, since $L / R \ll T_{s}$. We assume that $\hat{i}_{L}\left(T_{s}\right)=0$.

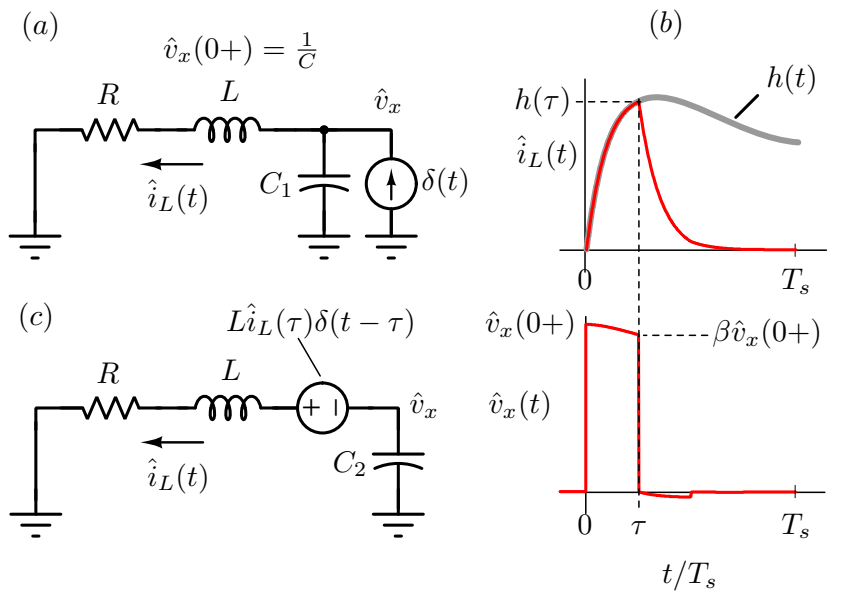

Fig. 15. (a) Equivalent circuit for the duration $0 \leq t<\tau$. (b) $\hat{i}_{L}(t)$ in response to an impulse current injected into the capacitor is denoted by $h_{L}(t)$. The capacitor voltage at $t=\tau-$ is $\beta \hat{v}_{x}(0)$. (c) Calculating the effect of the inductor current on the capacitor voltage during $\tau \leq t<2 \tau$.
Fig. 15(a) shows the equivalent circuit for the duration $0 \leq t<\tau$. In the analysis that follows, we denote the impulse response from the current injected into the capacitor to the inductor current $\hat{i}_{L}(t)$ by $h_{L}(t)$. In the Laplace domain, the transfer function can be seen to be

$$
\begin{aligned}
\frac{\hat{I}_{L}(s)}{\hat{I}_{i n}(s)} & =\mathcal{L}\left(h_{L}(t)\right) \\
& =\frac{1}{1+s C R+s^{2} L C} \equiv \frac{1}{\left(1+s \tau_{1}\right)\left(1+s \tau_{2}\right)} .
\end{aligned}
$$

Expressing the denominator polynomial as a product of two real poles is justified in the passive-mixer mode ${ }^{4}$, where $R C \gg L / R$. Without loss of generality, we assume that $\tau_{2}>\tau_{1}$. With this assumption, to first-order, $\tau_{1} \approx L / R$ and $\tau_{2} \approx R C$. It turns out that the following are better approximations.

$$
\tau_{1} \approx \frac{L}{R}\left(1+\frac{L}{C R^{2}}\right), \tau_{2} \approx R C\left(1-\frac{L}{C R^{2}}\right) .
$$

$h_{L}(t)$ is seen to be

$$
h_{L}(t)=\frac{1}{\tau_{2}-\tau_{1}}\left(e^{-\frac{t}{\tau_{2}}}-e^{-\frac{t}{\tau_{1}}}\right) u(t) .
$$

The inductor current at $t=\tau$ is $\hat{i}_{L}(\tau)=h_{L}(\tau)$. The voltage across the capacitor at $t=\tau$ is given by

$$
\begin{aligned}
\hat{v}_{x}(\tau) & =\frac{1}{C}\left[1-\int_{0}^{\tau} h_{L}(t) d t\right] \\
& =\bar{C} \underbrace{\frac{1}{\left(\tau_{2} e^{-\frac{\tau}{\tau_{2}}}-\tau_{1} e^{-\frac{\tau}{\tau_{1}}}\right)}}_{\beta} \equiv \beta \hat{v}_{x}(0+) .
\end{aligned}
$$

In practice, $\beta$ will be slightly smaller than one. At $t=\tau$, the inductor is abruptly switched to $C_{2}$, which is uncharged. The inductor current $\hat{i}_{L}(\tau)$ can be modeled as a voltage impulse $L \hat{i}_{L}(\tau) \delta(t-\tau)$ in an initially relaxed RLC network, as shown in Fig. 15(c). We can exploit reciprocity to determine the effect of this voltage impulse on $\hat{v}_{x}$ at $t=2 \tau$ as follows. Recall that a current impulse across the capacitor at $t=0$ results in an inductor current $h_{L}(\tau)$ at $t=\tau$. Invoking reciprocity, a voltage impulse of amplitude $L h_{L}(\tau)$ at $t=\tau$ in series with the inductor should result in a capacitor voltage $-L h_{L}(\tau) \cdot h_{L}(\tau)=-L h_{L}^{2}(\tau)$ at $t=2 \tau$. Using $\hat{v}_{x}(0+)=1 / C$, this can be equivalently expressed as $-L C h_{L}^{2}(\tau) \hat{v}_{x}(0+)$. We determine $\hat{i}_{L}(2 \tau)$ next. Referring to Fig. 15(c), we see that the impulse response relating the voltage in series with the inductor (and capacitor) to $\hat{i}_{L}(t)$ is $C h_{L}^{\prime}(t)$. Since the magnitude of the impulse voltage is $L \hat{i}_{L}(\tau)$ and it occurs at $t=\tau$, it follows that

$$
\begin{aligned}
\hat{i}_{L}(2 \tau) & =-\left.L \hat{i}_{L}(\tau) C \frac{d h_{L}(t)}{d t}\right|_{t=\tau} \\
& =\underbrace{\hat{i}_{L}(\tau)}_{h_{L}(\tau)} \underbrace{\frac{\left(\tau_{1} e^{-\frac{\tau}{\tau_{2}}}-\tau_{2} e^{-\frac{\tau}{\tau_{1}}}\right)}{\left(\tau_{2}-\tau_{1}\right)}}_{\alpha} \equiv \alpha h_{L}(\tau) .
\end{aligned}
$$

${ }^{4}$ Note that the quality factor of this RLC network is smaller than 1 even for inductors as large as several $\mathrm{nH}$, due to the series resistance (typically $50 \Omega$ ) and the multi-pF $C$ values needed to achieve $N$-path filter bandwidths in the order of a few MHz targeted at channel selection. 
In practice, since $L / R<T_{s}$, it will follow that $\alpha$ will be a small number.

At $t=2 \tau$, the inductor is switched to $C_{3}$. Using the same line of arguments as above, it is easy to see that

$$
\hat{v}_{x}(3 \tau)=-L \alpha h^{2}(\tau), \hat{v}_{x}(4 \tau)=-L \alpha^{2} h^{2}(\tau)
$$

and

$$
\hat{i}_{L}(3 \tau)=\alpha^{2} h_{L}(\tau), \hat{i}_{L}(4 \tau)=\alpha^{3} h_{L}(\tau) .
$$

The table of Fig. 14(a) gives the inductor current and capacitor voltages at integer multiples of $\tau$. The last row is of particular interest, as it allows us to write a recursive relation for $\hat{i}_{L}(t)$. Since $\alpha \ll 1$ for practical values of $L$, we assume that $\hat{i}_{L}(4 \tau)=\hat{i}_{L}\left(T_{s}-\right)=0$. Like we did when we analyzed the structure with a parasitic source capacitance, we can write

$$
\begin{aligned}
\hat{i}_{L}(t)= & p(t)+\beta \hat{i}_{L}\left(t-T_{s}\right)-L C h_{L}^{2}(\tau) \hat{i}_{L}\left(t-T_{s}-\tau\right) \\
& -\alpha L C h_{L}^{2}(\tau) \hat{i}_{L}\left(t-T_{s}-2 \tau\right) \\
& -\alpha^{2} L C h_{L}^{2}(\tau) \hat{i}_{L}\left(t-T_{s}-3 \tau\right)
\end{aligned}
$$

where $p(t)$ denotes $\hat{i}_{L}(t)$ for $0 \leq t<T_{s}$.

Since $H_{e q}(f)$ is the Fourier transform of $\hat{i}_{L}(t)$, we have

$$
H_{e q}(f)=\frac{P(f)}{\left[\begin{array}{c}
1-\beta z^{-4}+L C h_{L}^{2}(\tau) z^{-5} \\
+\alpha L C h_{L}^{2}(\tau) z^{-6}+\alpha^{2} L C h_{L}^{2}(\tau) z^{-7}
\end{array}\right]}
$$

where $z \equiv e^{j 2 \pi f \tau}$. A few observations are in order.

a. Adding the inductor results in a $\beta$ that is closer to unity than when $L=0$. While this can be deduced from (17), this makes intuitive sense due to the following. Adding the inductor reduces current drawn from $C_{1}$, causing a larger fraction of its charge to remain on it at $t=\tau$. Thus, we should expect that the addition of the series inductor increases the magnitude of $H_{e q}(f)$ around multiples of $f_{s}$.

b. The sign of the coefficient of $z^{-5}$ in the denominator of (22) is positive, unlike in the case of a parasitic source capacitor. Thus, we should expect that the peak of the response around $f_{s}$ shifts to the right, while that around $3 f_{s}$ shifts towards the left. The peak around $2 f_{s}$ does not shift. From (22), it is easy to see that the peak shift around $f_{s}$ is given by

$$
\frac{\Delta f}{f_{s}} \approx \frac{L C h^{2}(\tau)}{2 \pi \beta} .
$$

c. In our analysis, we expressed the transfer function of the RLC circuit as the product of two first-order systems with time-constants $\tau_{1}$ and $\tau_{2}$. This is justified in practice, since $R C \gg L / R$.

d. When considering analysis with a parasitic source capacitance $C_{s}$, or series inductance $L, \beta$ represents the fractional loss of charge on the filter capacitor in a timeinterval $\tau . \alpha$ represents the coupling from one filter capacitor to the next, induced by $C_{s}$ or $L$.

A question that remains is "how do we determine $p(t)$ ?". Recall that $p(t)$ is $\hat{i}_{L}(t)$ in the interval $0 \leq t<T_{s}$ when a current impulse $\delta(t)$ is injected into $C_{1}$ in the circuit of Fig. 14(a). Due to the action of the switches, the $\hat{v}_{x}$ goes to (a)

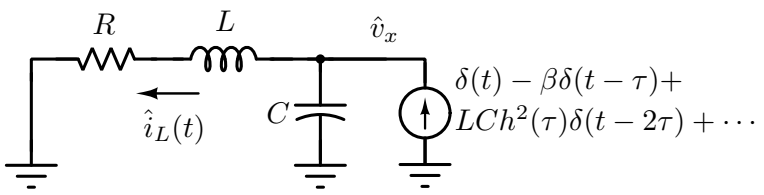

(b)

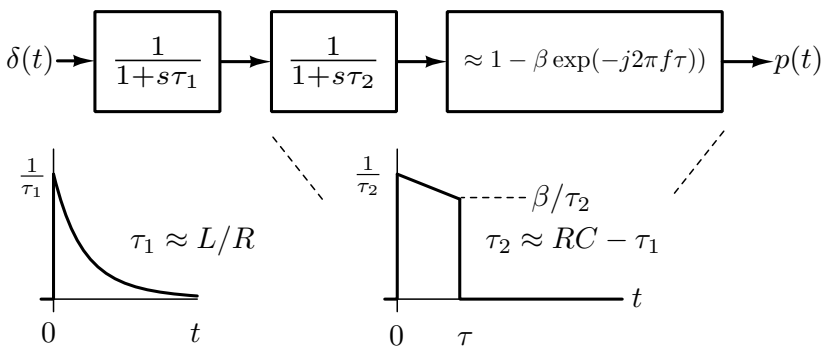

Fig. 16. (a) Approach to determine $p(t)$. (b) The inductor effectively prefilters the input with an $R L$ lowpass filter.

zero at $t=\tau+, 2 \tau+$ and $3 \tau+. p(t)$ can be thought of as the current that results by exciting the capacitor of an LTI RLC circuit with a impulse current sequence as shown in Fig. 16(a). The sequence is chosen so that $\hat{v}_{x}(t)$ goes to zero at $t=\tau, 2 \tau, 3 \tau$, like in the $N$-path circuit of Fig. 14(a). Since we know $\hat{v}_{x}$ at $\tau, 2 \tau$ and $3 \tau$ is given by $\beta / C,-L h_{L}^{2}(\tau)$ and $-\alpha L h_{L}^{2}(\tau)$ respectively, it is seen that $\hat{i}(t)$ in Fig. 16(a) should be

$$
\begin{aligned}
\hat{i}(t)= & \delta(t)-\beta \delta(t-\tau)+L C h_{L}^{2}(\tau) \delta(t-2 \tau) \\
& +\alpha L C h_{L}^{2}(\tau) \delta(t-3 \tau) .
\end{aligned}
$$

Since $h_{L}(t)$ denotes the inductor current in response to a current impulse injected into the capacitor, it follows that $p(t)$, which is $\hat{i}_{L}(t)$ in response to $i(t)$ above, is given by

$$
\begin{aligned}
p(t)= & h_{L}(t)-\beta h_{L}(t-\tau)+L C h_{L}^{2}(\tau) h_{L}(t-2 \tau) \\
& +\alpha L C h_{L}^{2}(\tau) h(t-3 \tau) .
\end{aligned}
$$

In the frequency domain, this corresponds to

$P(f)=H(f)\left(1-\beta z^{-1}+L C h_{L}^{2}(\tau) z^{-2}+\alpha L C h_{L}^{2}(\tau) z^{-3}\right)$.

Simplifying $P(f)$ above results in an intuitively appealing result, developed below. Neglecting the $z^{-2}$ and $z^{-3}$ terms (since they are much smaller than $\beta$ ), we can express $P(f)$ as

$$
P(f) \approx H(f)\left(1-\beta z^{-1}\right)=\frac{1-\beta z^{-1}}{\left(1+j 2 \pi f \tau_{1}\right)\left(1+j 2 \pi f \tau_{2}\right)}
$$

which when drawn in block diagram form, is as shown in Fig. 16(b). It is instructive to determine the impulse response of the second and third blocks in cascade. Since $\tau_{2}(\approx R C) \gg T_{s}$, the impulse response of the cascade is an exponentially decaying pulse that lasts for a duration of $\tau$. This is the shape of $p(t)$ that would be seen, had $L$ been zero. However, this is pre-filtered by a first order transfer function with time-constant $\tau_{1} \approx L / R$, caused by the lowpass action of the $R L$-series network. As a result, $p(t)$ is much "smoother" that it would otherwise be, resulting in a better attenuation at high frequencies. Further, the reasoning 
above suggests that the 3-dB bandwidth of the RL low-pass filter, namely $R /(2 \pi L)$, should be chosen to be higher than the input frequency $\approx f_{s}$, to prevent the desired input from being filtered out. This is consistent with the heuristic reasoning in [26]. To summarize,

a. Introducing the series inductor increases the gain of $H_{e q}(f)$ around $f_{s}$, thanks to a $\beta$ which is closer to one (when compared to $\beta$ without the inductor). This results in a higher peak gain around multiples of $f_{s}$, as well as a higher $\mathrm{Q}$, since the impulse response lasts longer.

b. The inductor acts as a pre-filter, by introducing an $R L$ lowpass filter into $H_{e q}(f)$. This is beneficial, since frequencies at higher multiples of $f_{s}$ are better attenuated before downconversion. The bandwidth of this low-pass filter must be chosen so as to let through the desired signal (with frequency $\approx f_{s}$ ).

c. The peak of $H_{e q}(f)$ around $f_{s}$ is shifted to the right, i.e., a frequency greater than $f_{s}$, by an amount given by (23). The root cause for the frequency shift, as seen from (22), is largely the voltage created on $C_{2}$ due to the coupling introduced by the inductor.

d. The model for the total voltage across the capacitor in the passive-mixer mode can be thought of as zeroorder-holding the sampled voltage on the capacitor for a complete clock period, as in Fig. 10(e).

- $\quad$ Spectre PXF of entire waveform

$-\left|H_{e q}(f)\right|$ (analytical)

$-\left|H_{e q}(f)\right|$ (simulated, sampled PXF)
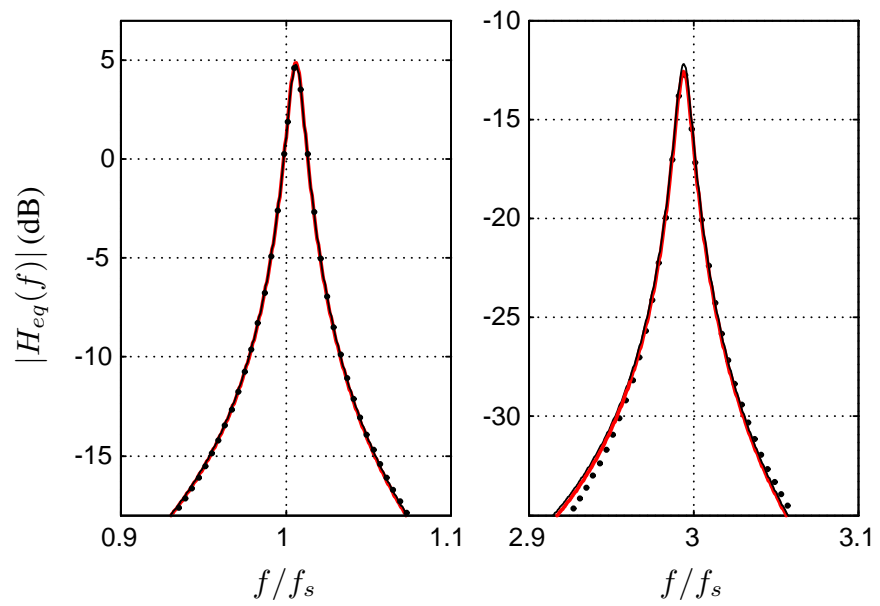

Fig. 17. Simulated $\left|H_{e q}(f)\right|$ compared with analytical results for $N=4$, $f_{s}=1 \mathrm{GHz}, C=50 \mathrm{pF}, R=50 \Omega$ and $L=10 \mathrm{nH}$. The periodic transfer function of the entire voltage waveform across $C_{1}$ is also shown.

Fig. 17 compares the simulated $\left|H_{e q}(f)\right|$ with our analytical results. Similar curves were generated for different $L$ values up to $10 \mathrm{nH}$, where the maximum model deviation was found to be less than $0.5 \mathrm{~dB}$ for the $L=10 \mathrm{nH}$ case. The mixer used $N=4, f_{s}=1 \mathrm{GHz}, C=50 \mathrm{pF}, R=50 \Omega$ and $L=10 \mathrm{nH}$. The inductance chosen is somewhat large, since the $3-\mathrm{dB}$ bandwidth of the LR lowpass filter is only $R /(2 \pi L)=800 \mathrm{MHz}$. The peak gain around $f=3 f_{s}$ determined using (22) deviates from the simulated value by about $0.4 \mathrm{~dB}$. This is because at this value of $L, \hat{i}_{L}\left(T_{s}\right)$ is not negligible when compared to $\hat{i}_{L}(\tau)$. The simulated $\left|H_{e q}(f)\right|$ matches well with the response obtained from periodic transfer function (PXF) simulations of the entire waveform (not just the sampled output). This indicates that the sampled voltage on $C_{1,2,3,4}$ plays the most crucial role in determining the eventual output of the mixer (which will be generated by harmonic combination of the waveforms across $C_{1,2,3,4}$.)

\section{PEAK-ShIFt FreE PASSIVE MiXers}

In the preceding sections, we saw that for $N=4$, a parasitic source capacitance causes the peaks of the magnitude response around $f_{s}$ to shift left, while adding an inductor in series causes the peak frequency to shift right. We saw that the root cause of this was the voltage sampled on the second capacitor due to the coupling effect of the inductor and parasitic source capacitor. Further we saw that the signs of the voltages induced on to the second capacitor are opposite for the inductive and capacitive cases. This suggests that using an inductor and appropriately chosen capacitor should result in a cancellation of these effects, thereby resulting in a negligible peak-shift. As shown below, this is indeed the case. Fig. 18 shows the adjoint network for the case of the

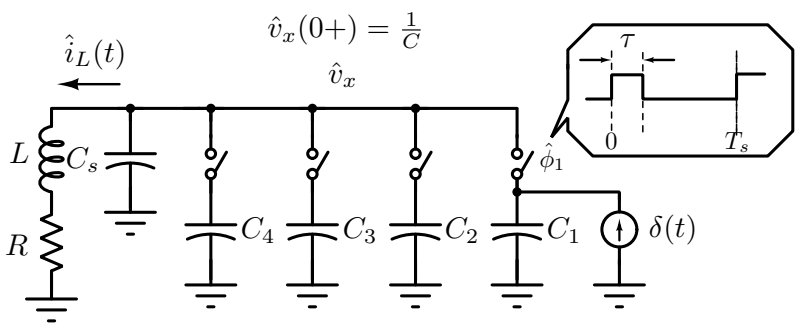

Fig. 18. Adjoint network for a 4-path system with series inductance and parasitic capacitance. $C_{s}$ can be chosen to ensure gain peaking at multiples of $f_{s}$.

inductor and parasitic capacitance both being present. Clearly, $\hat{v}_{x}(0+)=1 /\left(C+C_{s}\right)$. At $t=\tau-, \hat{v}_{x}(\tau-)=\beta \hat{v}_{x}(0+)$, and $\hat{i}_{L}(\tau)=h_{L}(\tau)$. Note that $\beta$ and $h_{L}(\tau)$ must be determined using (17) and (16) using $\left(C+C_{s}\right)$ in place of $C$. When $C_{2}$ is switched, charge sharing occurs, resulting in $\hat{v}_{x}(\tau+)$ dropping to $\hat{v}_{x}(\tau-) C_{s} /\left(C+C_{s}\right)$. Using superposition and the arguments preceding (22) we see that $\hat{v}_{x}(2 \tau-)$ (which is the voltage stored on $C_{2}$ ) is given by

$$
\hat{v}_{x}(2 \tau-)=\beta \hat{v}_{x}(\tau+)-L h^{2}(\tau)=\frac{\beta^{2} C_{s}}{\left(C+C_{s}\right)^{2}}-L h^{2}(\tau) .
$$

This will be zero if $\beta^{2} C_{s}=\left(C+C_{s}\right)^{2} L h^{2}(\tau)$. Using this, (17) and (16), and approximating $\tau_{2} \approx R\left(C+C_{s}\right)$ and $\tau_{1} \approx L / R$, we have

$$
\frac{L}{C_{s}} \approx \frac{\beta^{2}}{h^{2}(\tau)\left(C+C_{s}\right)^{2}} \approx R^{2} \frac{1}{\left[1-e^{\left(\frac{\tau}{\tau_{2}}-\frac{\tau}{\tau_{1}}\right)}\right]} .
$$

From the expression above, it is seen that the characteristic impedance $\sqrt{L / C_{s}}$ should be chosen so that it is somewhat higher than $R$. 


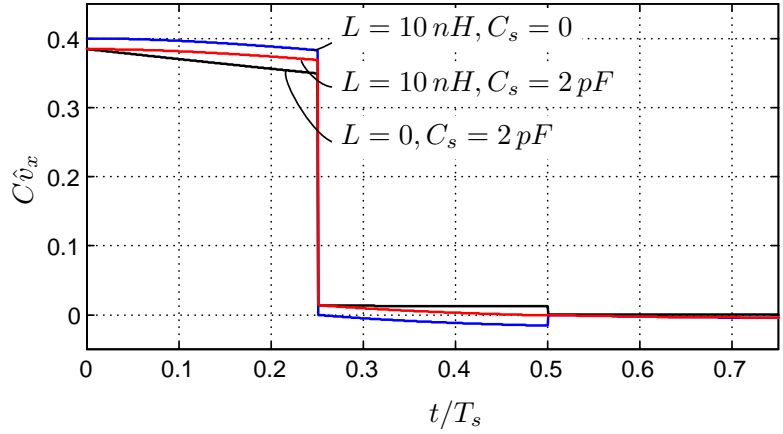

Fig. 19. $\hat{v}_{x}(t)$ for different choices of $L$ and $C_{s} . N=4, f_{s}=1 \mathrm{GHz}, C=$ $50 \mathrm{pF}, R=50 \Omega$.
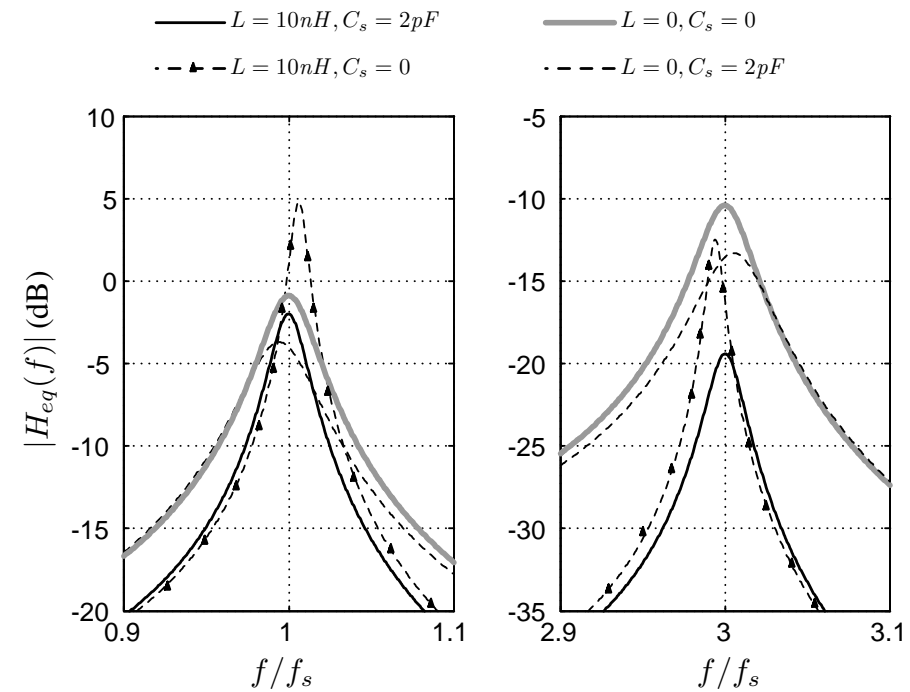

Fig. 20. Zoomed-in portions of the simulated $\left|H_{e q}(f)\right|$ around $f_{s}$ and $3 f_{s}$ for different choices of $L$ and $C_{s} . N=4, f_{s}=1 \mathrm{GHz}, C=50 \mathrm{pF}, R=50 \Omega$.

Figs. 19 and 20 confirm the intuition above. Fig. 19 shows $\hat{v}_{x}(t)$ (scaled by the numerical value of $C$ ) during the first clock cycle. With an inductive source impedance and $C_{s}=0$, we see that $\hat{v}_{x}$ goes negative when $C_{2}$ is switched, so that $\hat{v}_{x}(2 \tau-)$ is negative. On the other hand, when $L=0$ and $C_{s}=2 \mathrm{pF}, \hat{v}_{x}(2 \tau-)$ is positive due to charge sharing. Further, $\hat{v}_{x}(0+)$ is smaller than in the inductor-only case. With both the inductor and (appropriately chosen) capacitor in place, we see that their effects largely cancel each other, and $\hat{v}_{x}(2 \tau-) \approx 0$. Thus, we should expect that the response now peaks approximately at $f_{s}, 3 f_{s}$ etc. Fig. 20 shows the zoomed-in portions of the magnitude response around $f_{s}$ and $3 f_{s}$ for different choices of $L$ and $C_{s}$. Using a $10 \mathrm{nH}$ inductor alone not only results in a higher peak gain around $f_{s}$, but a peak that is shifted right. $C_{s}=2 \mathrm{pF}$ with $L=0$ results in a lower peak gain, as well as a peak that is shifted left. When $L=10 \mathrm{nH}$ and $C=2 \mathrm{pF}$, we see (as we expected) that the peaks in the responses occur approximately at $f_{s}$ and $3 f_{s}$.

\section{A. Effect of center-frequency tuning:}

Fig. 21 shows the simulated $\left|H_{e q}(f)\right|$ around $f_{s}$, as $f_{s}$ is swept from $250 \mathrm{MHz}$ to $1 \mathrm{GHz}$. Note that the $\mathrm{x}$-axis is normalized to $f_{s}$. When $L$ and $C_{s}$ are zero, we see that
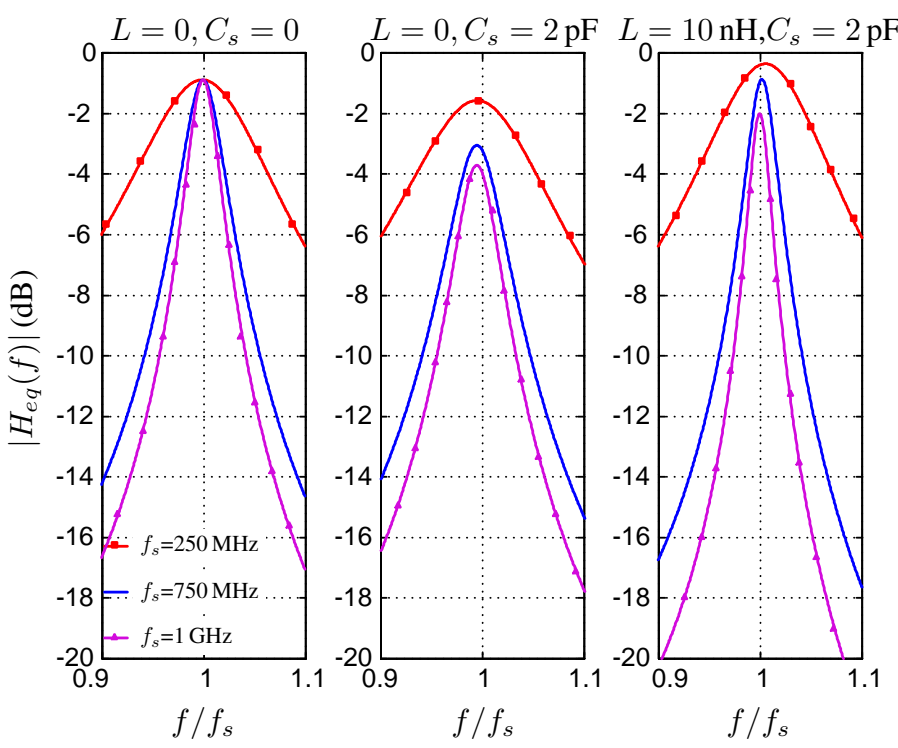

Fig. 21. Simulated effect of frequency variation on $\left|H_{e q}(f)\right|$ as $f_{s}$ is tuned from $250 \mathrm{MHz}$ to $1 \mathrm{GHz}$. The $\mathrm{x}$-axis is normalized to $f_{s}$.

the peak location and gain at $f_{s}$ remain virtually unchanged with tuning. With $C_{s}=2 \mathrm{pF}$, we see degradation in the gain and selectivity, as well as a peak shift. When the series inductor $\mathrm{L}$ is added to compensate for the effect of $\mathrm{Cs}$, this benefits gain and selectivity. The gain for low $f_{s}$ is enhanced (from what one would have obtained without $L$ and $C_{s}$ ), and gradually decreases as $f_{s}$ increases. The bandwidth of the $L C_{s} R$ network is about $1 \mathrm{GHz}$, so increasing $f_{s}$ beyond $1 \mathrm{GHz}$ yields no benefit.

\section{Conclusions}

We analyzed the effect of source capacitance and inductance on the performance of $N$-path structures using the adjoint-network approach of [21]. The use of the adjoint network not only simplified the algebra (when compared with conventional analysis based on [25]), but also gave useful insights on the influence of these "extra" elements on circuit performance. We showed that source inductance/capacitance introduce coupling between the capacitors of the $N$-path circuit. Parasitic capacitance degrades gain around $f_{s}$, and shifts the peak-gain frequency to the left of $f_{s}$. Source inductance, on the other hand, increases the gain around $f_{s}$, while shifting the peak-gain frequency to the right of $f_{s}$. Our analysis yielded simple expressions for the peak shift in terms of circuit parameters. Finally, we showed that an appropriate choice of inductance and capacitance can restore the peakfrequency to $f_{s}$, increase selectivity and reduce the gain of the $N$-path structure at higher multiples of $f_{s}$. Simulation results confirmed the validity of our analysis.

\section{REFERENCES}

[1] H. Darabi, A. Mirzaei, and M. Mikhemar, "Highly integrated and tunable RF front ends for reconfigurable multiband transceivers: A tutorial," IEEE Transactions on Circuits and Systems I: Regular Papers, vol. 58, no. 9, pp. 2038-2050, 2011. 
[2] E. Sacchi, I. Bietti, S. Erba, L. Tee, P. Vilmercati, and R. Castello, "A $15 \mathrm{~mW}, 70 \mathrm{kHz} 1 / f$ corner direct conversion CMOS receiver," in Proceedings of the IEEE Custom Integrated Circuits Conference. IEEE, 2003, pp. 459-462.

[3] Z. Ru, N. A. Moseley, E. A. Klumperink, and B. Nauta, "Digitally enhanced software-defined radio receiver robust to out-of-band interference," IEEE Journal of Solid-State Circuits, vol. 44, no. 12, pp. 33593375, 2009.

[4] D. Murphy, H. Darabi, A. Abidi, A. A. Hafez, A. Mirzaei, M. Mikhemar, and M.-C. F. Chang, "A blocker-tolerant, noise-cancelling receiver suitable for wideband wireless applications," IEEE Journal of SolidState Circuits, vol. 47, no. 12, pp. 2943-2963, 2012.

[5] H. Khatri, P. S. Gudem, and L. E. Larson, "Distortion in current commutating passive CMOS downconversion mixers," IEEE Transactions on Microwave Theory and Techniques, vol. 57, no. 11, pp. 2671-2681, 2009.

[6] S. Chehrazi, A. Mirzaei, and A. A. Abidi, "Second-order intermodulation in current-commutating passive FET mixers," IEEE Transactions on Circuits and Systems I: Regular Papers, vol. 56, no. 12, pp. 2556-2568, 2009.

[7] A. Mirzaei, H. Darabi, J. C. Leete, and Y. Chang, "Analysis and optimization of direct-conversion receivers with $25 \%$ duty-cycle currentdriven passive mixers," IEEE Transactions on Circuits and Systems I: Regular Papers, vol. 57, no. 9, pp. 2353-2366, 2010.

[8] M. Sosio, A. Liscidini, and R. Castello, "An intuitive current-driven passive mixer model based on switched-capacitor theory," IEEE Transactions on Circuits and Systems II: Express Briefs, vol. 60, no. 2, pp 66-70, 2013.

[9] E. S. Atalla, F. Zhang, P. T. Balsara, A. Bellaouar, S. Ba, and K. Kiasaleh, "Time-domain analysis of passive mixer impedance: A switchedcapacitor approach," IEEE Transactions on Circuits and Systems I: Regular Papers, vol. 64, no. 2, pp. 347-359, 2017.

[10] E. Klumperink, Z. Ru, N. Moseley, and B. Nauta, "Interference rejection in receivers by frequency translated low-pass filtering and digitally enhanced harmonic-rejection mixing," in Digitally-Assisted Analog and RF CMOS Circuit Design for Software-Defined Radio. Springer, 2011, pp. $113-150$.

[11] H. K. Subramaniyan, E. A. Klumperink, V. Srinivasan, A. Kiaei, and B. Nauta, "RF transconductor linearization robust to process, voltage and temperature variations," IEEE Journal of Solid-State Circuits, vol. 50, no. 11 , pp. 2591-2602, 2015.

[12] A. Molnar and C. Andrews, "Impedance, filtering and noise in $\mathrm{N}$ phase passive CMOS mixers," in Proceedings of the Custom Integrated Circuits Conference (CICC). IEEE, 2012, pp. 1-8.

[13] C. Andrews and A. C. Molnar, "A passive mixer-first receiver with digitally controlled and widely tunable RF interface," IEEE Journal of solid-state circuits, vol. 45, no. 12, pp. 2696-2708, 2010.

[14] _ "Implications of passive mixer transparency for impedance matching and noise figure in passive mixer-first receivers," IEEE Transactions on Circuits and Systems I: Regular Papers, vol. 57, no. 12, pp. 30923103, 2010.

[15] C. Andrews, C. Lee, and A. Molnar, "Effects of LO harmonics and overlap shunting on N-phase passive mixer based receivers," in Proceedings of the ESSCIRC (ESSCIRC). IEEE, 2012, pp. 117-120.

[16] H. Westerveld, E. Klumperink, and B. Nauta, "A cross-coupled switchRC mixer-first technique achieving $+41 \mathrm{dBm}$ out-of-band IIP3," in Proceedings of the Radio Frequency Integrated Circuits Symposium. IEEE, 2016, pp. 246-249.

[17] C.-k. Luo, P. S. Gudem, and J. F. Buckwalter, "0.4-6 GHz, 17-dBm $\mathrm{B} 1 \mathrm{~dB}, 36-\mathrm{dBm}$ IIP3 channel-selecting, low-noise amplifier for SAWless 3G/4G FDD receivers," in Radio Frequency Integrated Circuits Symposium (RFIC), 2015 IEEE. IEEE, 2015, pp. 299-302.

[18] Y. Lien, E. Klumperink, B. Tenbroek, J. Strange, and B. Nauta, "A highlinearity CMOS receiver achieving $+44 \mathrm{dBm}$ IIP3 and $+13 \mathrm{dBm} 1 \mathrm{~dB}$ for SAW-less LTE radio," in Proc. of the IEEE International Solid-State Circuits Conference (ISSCC),. IEEE, 2017, pp. 412-413.

[19] Y. Xu and P. R. Kinget, "A switched-capacitor RF front end with embedded programmable high-order filtering," IEEE Journal of SolidState Circuits, vol. 51, no. 5, pp. 1154-1167, 2016.

[20] A. Ghaffari, E. A. Klumperink, M. C. Soer, and B. Nauta, "Tunable high$Q N$-path band-pass filters: Modeling and verification," IEEE Journal of Solid-State Circuits, vol. 46, no. 5, pp. 998-1010, 2011.

[21] S. Pavan and E. Klumperink, "Simplified unified analysis of switchedRC passive mixers, samplers, and $N$-path filters using the adjoint network," IEEE Transactions on Circuits and Systems: Regular Papers, vol. 64, no. 11, p. to appear, 2017.
[22] M. C. Soer, E. A. Klumperink, P.-T. De Boer, F. E. Van Vliet, and B. Nauta, "Unified frequency-domain analysis of switched-series- $R C$ passive mixers and samplers," IEEE Transactions on Circuits and Systems I: Regular Papers, vol. 57, no. 10, pp. 2618-2631, 2010.

[23] T. Iizuka and A. A. Abidi, "FET-RC Circuits: A unified treatment - Part I: Signal transfer characteristics of a single-path," IEEE Transactions on Circuits and Systems I: Regular Papers, vol. 63, no. 9, pp. 1325-1336, 2016.

[24] — - "FET-RC Circuits: A unified treatment - Part II: Extension to multi-paths, noise figure, and driving-point impedance," IEEE Transactions on Circuits and Systems I: Regular Papers, vol. 63, no. 9, pp. 1337-1348, 2016.

[25] T. Strom and S. Signell, "Analysis of periodically switched linear circuits," IEEE Transactions on Circuits and Systems, vol. 24, no. 10, pp. 531-541, 1977.

[26] L. Duipmans, R. E. Struiksma, E. A. Klumperink, B. Nauta, and F. E. van Vliet, "Analysis of the signal transfer and folding in $N$-path filters with a series inductance," IEEE Transactions on Circuits and Systems I: Regular Papers, vol. 62, no. 1, pp. 263-272, 2015.

[27] A. El Oualkadi, M. El Kaamouchi, J.-M. Paillot, D. VanhoenackerJanvier, and D. Flandre, "Fully integrated high- $Q$ switched capacitor bandpass filter with center frequency and bandwidth tuning," in Proceedings of the Radio Frequency Integrated Circuits (RFIC) Symposium. IEEE, 2007, pp. 681-684.

[28] S. Pavan and R. S. Rajan, "Interreciprocity in linear periodically timevarying networks with sampled outputs," IEEE Transactions on Circuits and Systems II: Express Briefs, vol. 61, no. 9, pp. 686-690, 2014.

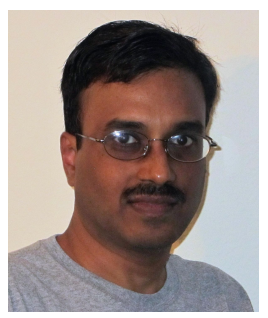

Shanthi Pavan obtained the B.Tech degree in Electronics and Communication Engg from the Indian Institute of Technology, Madras in 1995 and the M.S and Sc.D degrees from Columbia University, New York in 1997 and 1999 respectively. From 1997 to 2000, he was with Texas Instruments in Warren, New Jersey, where he worked on high speed analog filters and data converters. From 2000 to June 2002, he worked on microwave ICs for data communication at Bigbear Networks in Sunnyvale, California. Since July 2002, he has been with the Indian Institute of Technology-Madras, where he is now a Professor of Electrical Engineering. His research interests are in the areas of high speed analog circuit design and signal processing.

Dr. Pavan is the recipient of the IEEE Circuits and Systems Society Darlington Best Paper Award (2009), the Shanti Swarup Bhatnagar Award (2012) and the Swarnajayanthi Fellowship (2009) (from the Government of India), the Mid-career Research Excellence Award and the Young Faculty Recognition Award from IIT Madras (for excellence in teaching), the Technomentor Award from the India Semiconductor Association and the Young Engineer Award from the Indian National Academy of Engineering (2006). He is the author of Understanding Delta-Sigma Data Converters (second edition, with Richard Schreier and Gabor Temes). Dr. Pavan has served as the Editorin-Chief of the IEEE Transactions on Circuits and Systems: Part I - Regular Papers, and on the editorial boards of both parts of the IEEE Transactions on Circuits and Systems. He has served on the technical program committee of the International Solid State Circuits Conference, and been a Distinguished Lecturer of the Solid-State Circuits Society. He is a fellow of the Indian National Academy of Engineering. 


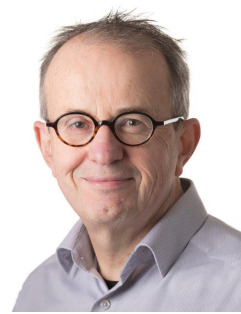

Eric Klumperink was born on April 4th, 1960, in Lichtenvoorde, The Netherlands. He received the B.Sc. degree from HTS, Enschede (1982), worked in industry on digital hardware and software, and then joined the University of Twente in 1984, shifting focus to analog CMOS circuit research. This resulted in several publications and his Ph.D. thesis "Transconductance Based CMOS Circuits" (1997). In 1998, Eric started as Assistant Professor at the ICDesign Laboratory in Twente and shifted research focus to RF CMOS circuits (e.g. sabbatical at the Ruhr Universitaet in Bochum, Germany). Since 2006, he is an Associate Professor, teaching Analog \& RF IC Electronics and guiding $\mathrm{PhD}$ and MSc projects related to RF CMOS circuit design with focus on Software Defined Radio, Cognitive Radio and Beamforming. He served as an Associate Editor for the IEEE TCAS-II (2006-2007), IEEE TCAS-I (2008-2009) and the IEEE JSSC (2010-2014), as IEEE SSC Distinguished Lecturer (2014/2015), and as member of the technical program committees of ISSCC (2011-2016) and the IEEE RFIC Symposium (2011-..). He holds several patents, authored and co-authored $150+$ internationally refereed journal and conference papers, and was recognized as 20+ ISSCC paper contributor over 1954-2013. He is a corecipient of the ISSCC 2002 and the ISSCC 2009 "Van Vessem Outstanding Paper Award". 